Article

\title{
A Near-Far-Field Model for Bubbles Influenced by External Electrical Fields
}

\author{
Juergen Geiser ${ }^{1, *(D)}$ and Paul Mertin ${ }^{2}(\mathbb{D}$ \\ 1 The Institute of Theoretical Electrical Engineering, Ruhr University of Bochum, Universitätsstrasse 150, \\ D-44801 Bochum, Germany \\ 2 Computational Electronics and Photonics, University of Kassel, Wilhelmshöher Allee 71, D-34121 Kassel, \\ Germany; paul.mertin@uni-kassel.de \\ * Correspondence: juergen.geiser@ruhr-uni-bochum.de
}

Received: 9 September 2019; Accepted: 31 October 2019; Published: 5 November 2019

check for updates

\begin{abstract}
In this paper, we present a model that is based on near-far-field charged bubble formation and transportation in an underlying dielectric liquid. The bubbles are controlled by the dielectric liquid, which is influenced by an external electrical field. This allows us to control the shape and volume of the bubbles in the dielectric liquid, such as water. These simulations are important to close the gap between the formation of charged bubbles, which is a fine-scale model and their transport in the underlying liquid, which is a coarse-scale model. In the fine-scale model, the formation of the bubbles and their influence of the electric-stress is approached by a near-field model, which is done by the Young-Laplace equation plus additional force-terms. In the coarse-scale model, the transport of the bubbles is approached by a far-field model, which is done with a convection-diffusion equation. The models are coupled with a bubble in cell scheme, which interpolates between the fine and coarse scales of the different models. Such a scale-dependent approach allows us to apply optimal numerical solvers for the different fine and coarse time and space scales and help to foresee the fluctuations of the charged bubbles in the E-field. We discuss the modeling approaches, numerical solver methods and we present the numerical results for the near-far-field bubble formation and transport model in a dielectric carrier fluid.
\end{abstract}

Keywords: bubble formation; dielectric fluids; electrical field; scale-dependent models; near-far-field approach; Young-Laplace equation; convection-diffusion equation; level-set method; coupling analysis

MSC: 35K25; 35K20; 74S10; 70G65

\section{Introduction}

We are motivated to model bubble formation and transport in dielectric liquids, which are applied in controlled production of gas/plasma bubbles in chemical, petrochemical, plasma or biomedical processes, see [1-4].

The benefit of additional particles or charged-bubbles in the dielectric carrier fluids are that they can influence their fluid behavior or have additional reactions in the fluid, see [5] or [6]. Therefore, the carrier or background liquid can be influenced in the following directions:

- $\quad$ The charged bubbles can be used as plasma bubbles and can be discharged, such applications can then be used to clean a polluted fluid, see [7].

- The charged bubbles can influence the flow of the underlying liquid, such applications can then be used in designing chemical reactors, see $[6,8]$. 
In our model, we concentrate on the first applications, we model the influence of the bubbles in a dielectric carrier fluid, such as water. Our modeling approach is based on a decomposition into scale-dependent models:

- Formation of bubbles (near-field model): the fast-fluctuating bubbles are formed with a near-field model and are influenced by the electric-stress related to the dielectric liquid, [9-11].

- Transport of bubbles (far-field model): the slow-fluctuating bubbles are transported with a far-field model, [12,13].

Here, we can assume that the fast-fluctuations of the formations and the slow-fluctuations of the transport of the bubble in the liquid carrier fluid has a clear spectral gap, which can be overcome with interpolating (e.g., spline-functions) between the different models, see [14]. Therefore, we apply a so-called modified particle in cell (PIC) method, see [14] and interpolate between the fine-model (microscopic model) and the coarse-model (macroscopic model). The benefit of such a decomposition allows us to simulate the two separate models independently, while we only couple them with the interpolation functions, see [15]

We also deal with numerical methods that take into account the different scale-dependent model. The formation process of bubbles are done with a microscopic model, the most standard solvers are done in the following:

- Fast ordinary differential equation (ODE) solvers for so-called bubble shape models, which are based on Young-Laplace equations: We apply the Young-Laplace equations, which are a system of ODEs. The benefit is that the solvers are very fast, while the drawback is that we are restricted to quasi-static processes, see [11].

- Coupled partial differential equation (PDE) solvers for so-called free surface models, which are based on Navier-Stokes equations with kinematic boundary condition. Here, we have to solve a free surface problem with PDE solvers. The benefit is that we can apply the model to instationary processes, the drawback is that we need to solve a system of PDEs, which is more time consuming, see [16].

The macroscopic model is done with a transport model based on an advection equation, where the most standard solver approaches are:

- Volume-of-fluid (VOF) methods: the VOF function presents the fraction of the volume in the grid cells, which is occupied by one of the two fluids. This is modeled with an advection equation. The benefit of the methods are mass conservation, the drawback of the methods are the problem to solve the sharp change in interface-region, see $[17,18]$.

- Level-set (LS) methods: the level set function presents a signed distance, which is positive on one fluid side and negative on the other fluid side, see [13]. It is also modeled with the same advection equation. The benefit of the methods is that we can solve if with fast high-speed flow methods, such as total variational diminishing (TVD) or essentially non-oscillatory (ENO) methods, see $[19,20]$. The drawback is the lack of mass conservation.

Combining the LS and VOF methods can also provide many benefits, although they are delicate to implement, see [18]. Here, we concentrate on the fast LS methods and take into account that we have to reinitialize or apply only small distance of the transport to reduce the mass-conservation error, see [18].

Such decomposition allows us to choose the optimal discretization and solver methods, which means that we can apply fast ODE-solvers for the near-field model and fast level-set methods for the far-field model.

The rest of this paper is organized as follows. The modeling problems and their solvers are presented in Section 2. The near-field solver is discussed in Section 3. In Section 4, we discuss the far-field Solver. The coupling of the models are discussed in Section 5 The numerical experiments are presented in Section 6. In Section 7, we summarize our results. 


\section{Mathematical Model}

Our mathematical model is based on a laboratory experiment, where gas bubbles are formed in a liquid and are transported after the formation process, see the plasma-experiment in [2]. The experiment is given as a thin capillary, where the gas bubbles are streamed in an homogeneous form and transported in a tube that is filled with liquid, see the Figure 1.

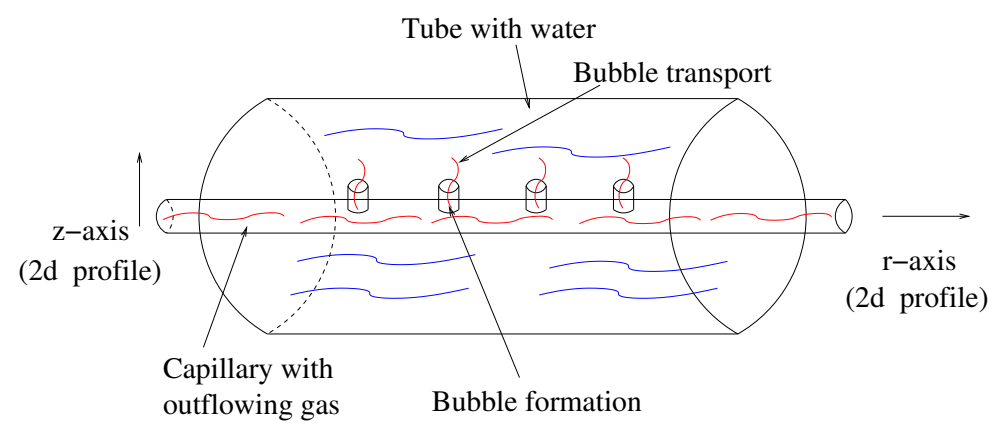

Figure 1. Sketch of our laboratory experiment (capillary with gaseous outflow into a tube filled with water).

We consider the profile of the tube and deal with the simplified approach of the experiment, which is given in Figure 2.

Tube with water and periodical gas-inflows
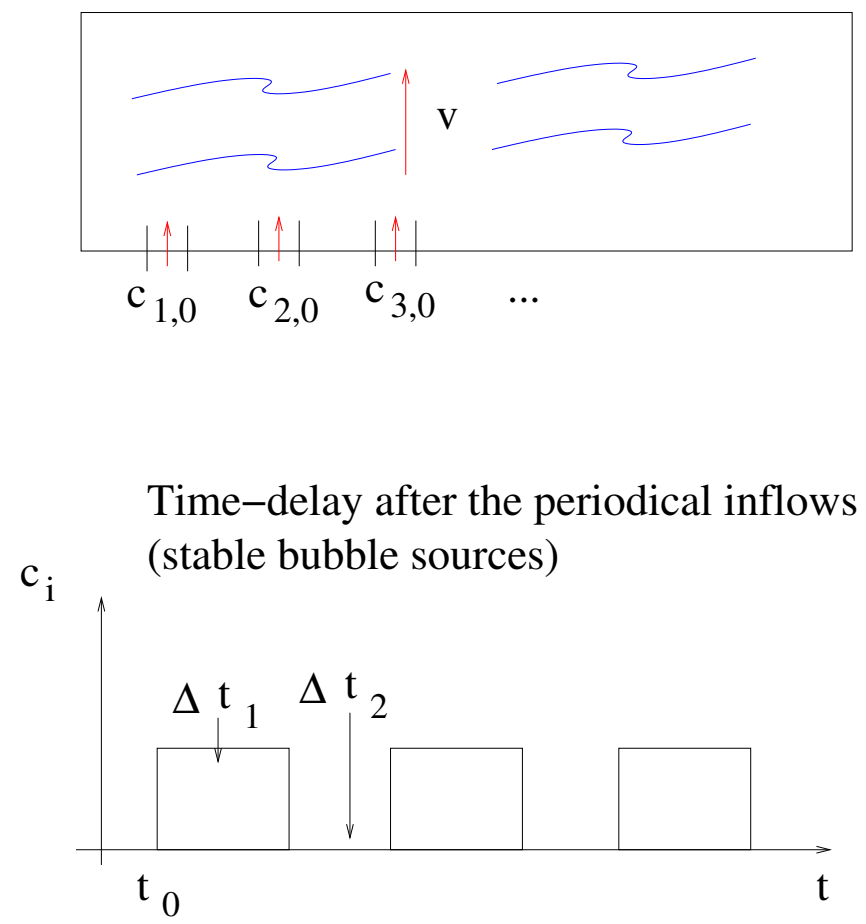

Figure 2. 2D model-reactor and inflow-conditions of the bubbles, in the upper figure, we present the 2D model of the reactor ( $\mathbf{v}$ is the velocity field, $c_{1,0}, \ldots, c_{M, 0}$ are the concentrations of the bubble inflow sources), in the lower figure, we present the concentration of the $i$-th gas bubble with periodically inflow ( $c_{i, 0}$ is the $i$-th concentration of the bubble inflow source, $t$ is the time, $t_{0}$ is the initial time, $\Delta t_{1}$ is the time-interval, where the source is switched on and $\Delta t_{2}$ is the time-interval, where the source is switched off). 
Furthermore, experiments are carried out in conjunction with electro-magnetic fields to influence the bubble formation and transport, see $[7,10]$. Here, the electric field is associated with the bubble oscillations, such as $[\mathrm{kHz}]$-frequency of the E-field is related to $[\mathrm{mm}]$-sized bubbles, see [10]. Based on the deforming and oscillation of the bubbles, while controlled with the frequency of the high voltage electrical field, we could achieve a shape, which is strategically more optimal (e.g., a small ellipsoid), see $[21,22]$, to enhance the E-field in the bubble. Then, the plasma ignition within the bubble gas can be done at reduced voltage, see [21] or [23]. These experiments are based on the following Figure 3, see also [7].

Bubbling reactors

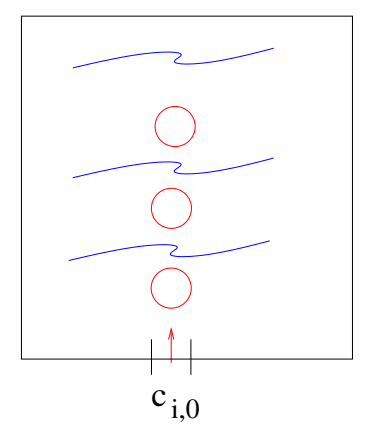

without E-field

(non-influenced

bubble-shapes)

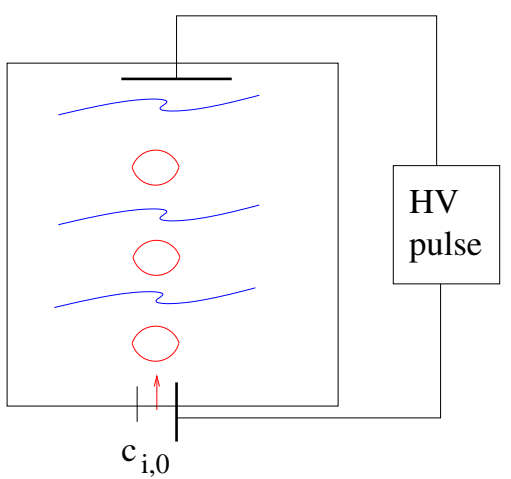

with E-field

(influenced

bubble-shapes)

Figure 3. Bubbling reactor: (left) we have non-influenced bubbles; (right) we have influenced bubbles based on an external high-voltage E-field.

Based on the decoupling of formation and transport, while we assume that the formation process is not influenced by the transport, see [24], we deal with two different decoupled models:

- Near-field approach, which is based on a Young-Laplace equation, see [11], where we have a static shape after the formation of the bubbles.

- Far-field approach, which is based on a convection-diffusion equation, see [25], where we have a rewriting into a level-set equation, such that we could transport the static bubble shapes, see [26] and [13].

The modelling approach is given in the following Figure 4.

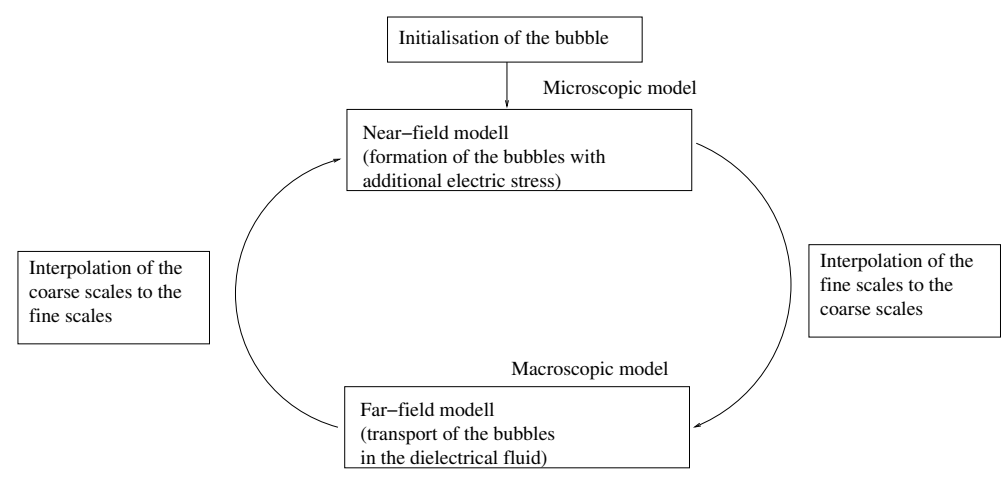

Figure 4. Modelling approach of the near-far-field coupling.

In the following, we discuss the different models. 


\subsection{Electrical-Field Approach for the Near-Field Model}

In the first step, we model the influence of the electrical-field in the near-field model.

Here, we are able to use the additional influence of the electrical field to the dielectric liquid, which can be modeled with the Maxwell stress tensor, see [27].

The electrical-field influences the normal stress or pressure $p_{E}$ of the underlying liquid, which is given as:

$$
p_{E}=(T \mathbf{n}) \cdot \mathbf{n},
$$

and $\mathbf{n}$ is the unit normal vector to the surface. The Maxwell-stress tensor $T$ is given as:

$$
T=\epsilon \mathbf{E} \otimes \mathbf{E}-\frac{1}{2} \epsilon(\mathbf{E} \cdot \mathbf{E}) \mathbf{I},
$$

where $\mathbf{E}$ is the electrical field and $\epsilon$ is the permittivity.

In the following, we assume a uniform electric field $\mathbf{E}_{0}$ and the relation of the normal stress or pressure of the bubble suspended in a dielectric liquid can be calculated with Equation (1) and can be given by, see [27]:

$$
p_{e} \approx \frac{9}{8} \epsilon\left|\mathbf{E}_{0}\right|^{2} \sin ^{2}(\Theta),
$$

where $\Theta$ is the angle of the elevation to the plane and we have a maximum with $\Theta=\pi / 2$, which means in the equatorial plane.

The additional pressure term based on the electrical field is used in the near-field model in the following Section 2.2.

\subsection{Near-Field Model}

The near-field model is discussed with respect to the formation of a drop or bubble, see [11,16].

The basic modeling idea is based on the so-called Young-Laplace equation, see [28]. We assumed to have quasi-static cases, while we had static pressure, and the surface tension forces were also effective elements, see [29].

The Young-Laplace-equation is derived by the balance of the liquid-gas interface, see [29], where we have:

$$
\Delta p+p_{e x}=\left(\frac{1}{r_{1}}+\frac{1}{r_{2}}\right) \sigma,
$$

where $\Delta p$ is the pressure-difference between the gas and liquid and $p_{e x}$ is an extra pressure; for example, in our case the electrical stress $p_{e x}=p_{e}$. Furthermore, $r_{1}$ and $r_{2}$ are the radii of the curvature, see Figure 5 , and $\sigma$ is the surface tension coefficient between the liquid and gas.

The parameters of the model and the geometry of the bubble are presented in Figure 5.

The parameterization of the free surface of the bubble in terms of the arclength $s$ can be modelled as a system of ordinary differential equation with a boundary value problems and is given as, see also [11,29]:

$$
\begin{aligned}
& \frac{d r}{d s}=\cos (\theta), \\
& \frac{d z}{d s}=\sin (\theta), \\
& \frac{d \theta}{d s}=\frac{\Delta p(z)}{\sigma}+\frac{p_{e}}{\sigma}-\frac{\sin (\theta)}{r},
\end{aligned}
$$


where the pressure difference is given as:

$$
\frac{\Delta p(z)}{\sigma}=\frac{2}{r_{0}}-\frac{g z}{\sigma}\left(\rho_{l}-\rho_{g}\right)
$$

where we have $r_{0}$ the radius of the curvature at the apex, $\sigma$ is the surface-tension coefficient, $\rho_{l}$ is the liquid density, $\rho_{g}$ is the gas density, $g$ is the gravity and $p_{e}$ the electrical stress.

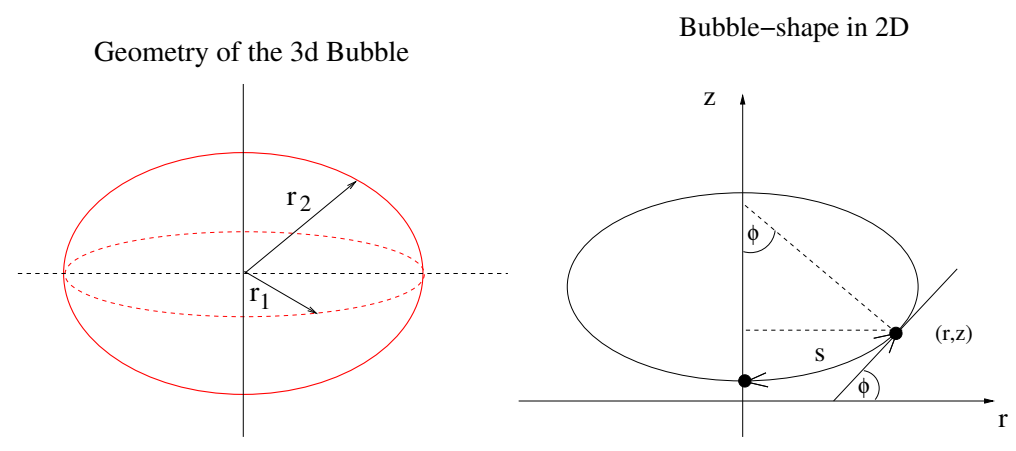

Figure 5. Bubble geometries: (left) $3 \mathrm{D}$ bubble with the $R_{1}$ the radius of the curvature in a horizontal section and $R_{2}$ the radius of the curvature in the vertical section, (right) set-up of the bubble shape in $2 \mathrm{D}$, where $\phi$ is the contact angle, $r$ is the horizontal length, $z$ is the vertical length and $s$ is the arclength along the free surface of the bubble.

In the following, we assume a small radius $r_{0} \leq 1$ and assume small vertical extensions; that is, $z \leq 1$. Then, we have a dominant $\frac{2}{R_{0}}$ term in the pressure difference, see also [29]:

$$
\frac{\Delta p(z)}{\sigma}=\frac{2}{r_{0}}-\frac{g z}{\sigma}\left(\rho_{l}-\rho_{g}\right) \approx \frac{2}{r_{0}} .
$$

Further, we have the boundary conditions, which are given as:

$$
\begin{aligned}
& r=a, z=0, \text { at } s=0, \\
& r=0, \frac{d z}{d s}=\frac{d z}{d r}=0, \text { at } s=L,
\end{aligned}
$$

where $L$ is the arc length of the bubble which is a-priori unknown and is numerically computed via the boundary value problem. Furthermore, we apply $a \rightarrow 0$, while $a$ is the initial radius of the bubble at $s=0$.

The ODE system (5)-(7) is a so-called boundary value problem, which can be solved with a multiple shooting methods, see also a discussion in Section 3 and the literature [30-32]. We apply a boundary-value solver of the MATLAB software-package, which is given as programmed in the function bop $4 c$. Based on the boundary values, we can solve the possible curvature of the bubble. We solve the axial-symmetric bubble, while it is sufficient to solve the half shape of the bubble. Then, we can measure the different diameters of the bubble ellipse and such results are interpolated on a grid, which is used in the far-field solver methods, see Section 5.

\subsection{Far-Field Approach}

For the far-field model, we base our work on macroscopic transport, see [18,33]. We assume that the flow is laminar and incompressible. Furthermore, we concentrate on the two-dimensional tube-problem, which is given in Figure 2. 
The conservation equation for the two fluids are given by the Navier-Stokes equation, which is also called the two-phase flow equation, see also [16]:

$$
\begin{aligned}
& \nabla \cdot \mathbf{v}=0,(x, y, t) \in \Omega \times[0, T], \\
& \rho\left(\frac{\partial \mathbf{v}}{\partial t}+\mathbf{v} \cdot \nabla \mathbf{v}\right)=-\nabla p+\nabla \cdot\left(\mu\left(\nabla \mathbf{v}+\nabla \mathbf{v}^{t}\right)\right)+\rho_{l} \mathbf{g}+\mathbf{F}_{\sigma},(x, y, t) \in \Omega \times[0, T],
\end{aligned}
$$

where $\mathbf{v}$ is the velocity vector, $\rho_{l}$ is the fluid density of the liquid, $\sigma$ is the surface tension of the liquid, $\mu$ is the fluid viscosity of the liquid and $\rho$ is the fluid density of the liquid. $\mathbf{g}$ is the gravitational acceleration and $\mathbf{F}_{\sigma}$ is the surface tension force. Furthermore, $\Omega \in \mathbb{R}^{2}$ is the underlying reactor-domain and $T \in \mathbb{R}^{+}$is the end-time of the simulations.

The detailed coordinates of the bubble reactor is given in Figure 6.

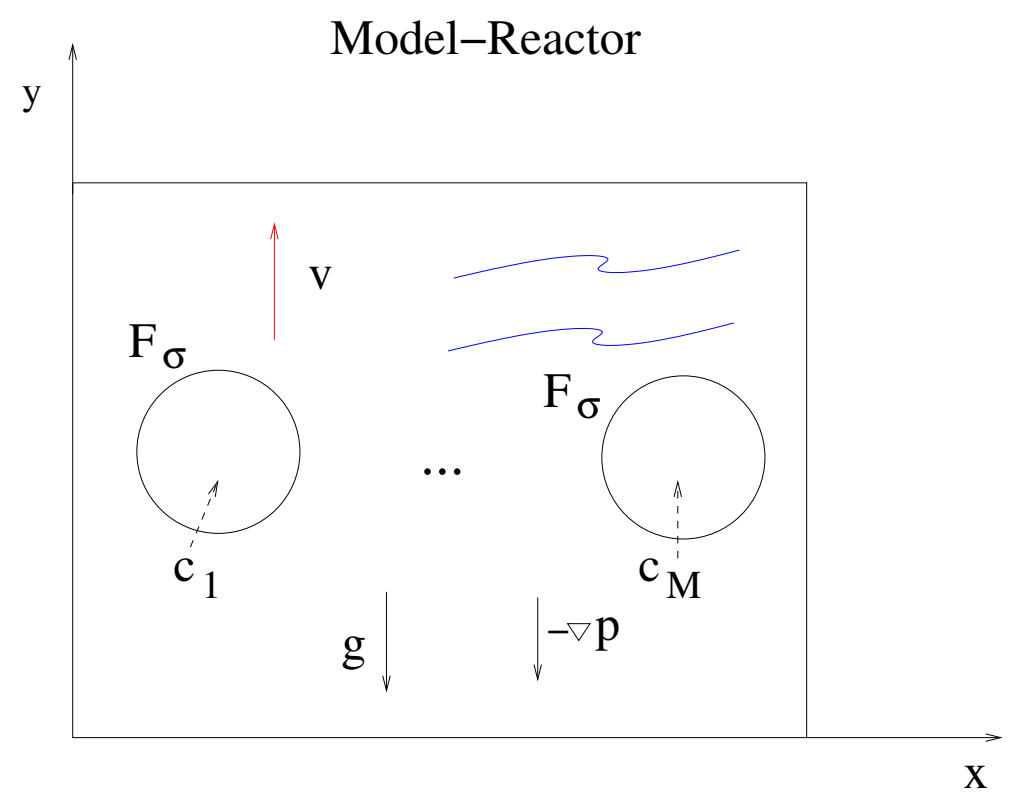

Figure 6. The coordinates of the model-reactor with the concentrations of the bubbles $c_{1}, \ldots, c_{M}$, the velocity $v$, the gravitational acceleration $g$, the pressure $p$ and the surface tension force $F_{\sigma}$.

The modeling equations of the gas bubble in the liquid is given by a transport equation, which is based on the idea of the volume of fluid and level set methods.

We assume the indicator functions $u_{i}(\mathbf{x}, t)$ with $i=1, \ldots, M$, while $M$ is the number of bubble sources.

We define the values of the function as:

$$
\left\{\begin{array}{cc}
u_{i}(\mathbf{x}, t)=1, & \text { gas-phase, } \\
u_{i}(\mathbf{x}, t)=0, & \text { liquid-phase, } \\
0<u_{i}(\mathbf{x}, t)<1, & \text { interface. }
\end{array}\right.
$$

Furthermore, we assume the absence of phase changes and then, we deal with the transport equation of the gas-phase as:

$$
\begin{aligned}
& \frac{\partial u_{i}}{\partial t}+\nabla\left(\mathbf{v} u_{i}\right)=0,(x, y, t) \in \Omega \times[0, T], i=1, \ldots, M, \\
& u_{i}(\mathbf{x}, 0)=u_{i, 0}(\mathbf{x}), \\
& i=1, \ldots, M
\end{aligned}
$$


where $\mathbf{v}$ is the velocity of the two-phase flow equation, and $u_{i}$ are the indicator functions of the gas-phases and $u_{i, 0}(\mathbf{x})$ are the initial indicator conditions.

In the following Assumption 1, we simplify the flow model in the bubbling reactor, such that we could concentrate on the transport-equations as far-field modeling equations.

Assumption 1. We deal with the following assumption to reduce the computational amount of the far-field model:

- We can assume a constant velocity in the reactor.

- We assume that the water and the gas are not interacting or change the constant velocity.

- We have such small velocities, such that the transport equations of the gas bubbles are sufficient, see [18].

- $\quad$ For such a simplification, we deal with $\mathbf{v}=\mathbf{v}_{0}=$ const in the transport Equations (15)-(17).

- The concentration of the bubbles are given as $c_{i}(\mathbf{x}, t)=c_{i, 0}(\mathbf{x}) u_{i}(\mathbf{x}, t)$, while $c_{i, 0}(\mathbf{x})$ is the initial concentration of the bubble.

Based on this assumption, we have the following benefits and drawbacks of the far-field modeling approach:

- Benefits:

- The model is simple and fast to compute.

- The model also allows us to discuss a dynamical shape.

- Drawbacks:

- The shape of the bubble is not preserved, while we assume a static shape.

- The influence of the speed of motion in the outer normal direction is not possible.

The far-field model can be solved with VOF- or LS-methods but we concentrate on the LS-methods to obtain highly accurate shapes of the bubbles. We discuss the solver in Section 4 .

\section{Near-Field Solver: System of Ordinary Differential Equations with Boundary Conditions}

For the near-field, we have to solve a BVP for ODEs, see [30-32].

We assume the following nonlinear ODE, given as:

$$
\begin{aligned}
& \frac{d \mathbf{y}}{d t}=\mathbf{f}(\mathbf{y}, t), t \in[a, b], \\
& B_{a} \mathbf{y}(a)+B_{b} \mathbf{y}(b)-\mathbf{d}=0,
\end{aligned}
$$

where $\mathbf{y}, \mathbf{d} \in \mathbb{R}^{n}, B_{a}, B_{b} \in \mathbb{R}^{n \times n}$ and $\mathbf{f}: \mathbb{R}^{n} \times \mathbb{R}^{+} \rightarrow \mathbb{R}^{n}$, see [32]. For example, we can rewrite our boudary value problem (5)-(7) into this vectorial notation.

The boundary value problem (18)-(19) can be solved with a multiple shooting algorithm, which is given in the following approach. We have a mesh $a=s_{1}<s_{2}<\ldots<s_{m}=b$, on each mesh interval $\left[s_{i}, s_{i+1}\right]$ with $1 \leq i \leq m-1$. Then, we apply the following steps to solve the boundary value problem (BVP).

We solve the BVP in the following five steps:

1. For the grid-points $\tilde{\mathbf{s}}_{1}, \ldots, \tilde{\mathbf{s}}_{m} \in \mathbb{R}^{n}$, we denote by $\mathbf{y}\left(s ; s_{j}, \tilde{\mathbf{s}}_{j}\right)$ the solution of the initial value problem

$$
\begin{aligned}
& \frac{d \mathbf{y}}{d t}=\mathbf{f}(\mathbf{y}, t), \\
& \mathbf{y}\left(s_{j} ; \tilde{\mathbf{s}}_{j}\right)=\tilde{\mathbf{s}}_{j} .
\end{aligned}
$$


2. We approach to the grid and define a piecewiese function $\tilde{\mathbf{y}}$ with

$$
\begin{aligned}
& \tilde{\mathbf{y}}(s)=\mathbf{y}\left(s ; s_{j}, \tilde{\mathbf{s}}_{j}\right), \text { for } s_{k} \leq s \leq s_{k+1}, 1 \leq j \leq m-1, \\
& \tilde{\mathbf{y}}\left(s_{m}\right)=\tilde{\mathbf{s}}_{m} .
\end{aligned}
$$

3. Then $\tilde{\mathbf{y}}$ solves the boundary value problem (18)-(19), if and only if:

$$
\begin{aligned}
& \mathbf{y}\left(s_{j+1} ; s_{j}, \tilde{\mathbf{s}}_{j}\right)=\tilde{\mathbf{s}}_{j+1}, \text { for } 1 \leq j \leq m-1, \\
& B_{a} \tilde{\mathbf{s}}_{1}+B_{b} \tilde{\mathbf{s}}_{m}-\mathbf{d}=0,
\end{aligned}
$$

4. Therefore, we have to solve the fixpoint problem, which is given by the initial value problem (20)-(21) and the conditions (24)-(25) and defined as:

$$
\mathbf{F}\left(\tilde{\mathbf{s}}_{1}, \ldots, \tilde{\mathbf{s}}_{m}\right)=0,
$$

which can be solved by linearization or nonlinear solver methods, e.g., Newton's method.

The multiple shooting algorithm allows us to compute the boundary value problem also with respect to the Neumann-boundary conditions. see [32]. The algorithm is a rewriting of the underlying BVP into an initial value problem (IVP) plus additional conditions. Based on the nonlinearity of the differential equation system, we have to apply linearization schemes or nonlinear solver schemes. In the Section 6.1, we apply the multiple shooting algorithm with linearization and additional solver schemes to approximate the nonlinear differential equation-system.

\section{Far-Field Solver: Level-Set Method}

We solve the transport Equations (15)-(17) with the level-set method, which allows us to follow the shapes of the bubble, see [13].

We reformulate the transport Equations (15)-(17) in the level-set equations, which are given as

$$
\begin{aligned}
& \frac{\partial u_{i}}{\partial t}=-F_{0}\left|\nabla u_{i}\right|,(\mathbf{x}, t) \in \Omega \times[0, T], \\
& u_{i}(\mathbf{x}, 0)=u_{i, 0}(\mathbf{x}), \\
& u_{i}(\mathbf{x}, t)=0.0,(\mathbf{x}, t) \in \partial \Omega \times[0, T], \\
& i=1, \ldots, M,
\end{aligned}
$$

where $\mathbf{v}$ is the constant velocity field with $\mathbf{v}=F_{0} \mathbf{n}$, while $\mathbf{n}=\nabla u_{i} /\left|\nabla u_{i}\right|$, see [13]. Furthermore, $\Omega$ is the computational domain and $T$ is the end time. The indicator functions are given as $u_{i}(\mathbf{x}, t)$ and the initialization $u_{i}(\mathbf{x}, 0)$ is done based on the results of the near-field computations, see Section 5.

These equations are well known as level-set equations and can be solved like convection-diffusion equations, see [13]. We concentrate on the benefits of the LS-methods, which are highly accurate and assume to deal with short transport-phases such that we could reduce the problem of the mass-conservation, see [18].

In the following, we apply the explicit different discretization methods in space, while we apply the level-set equation with the explicit time-discretization and apply upwind methods for the advection and outer normal direction term only in the $x$-direction, the same is also done with the $y$-direction. 
We discretize the Level-set equation with the explicit time-discretization and apply upwind methods for the advection and outer normal direction term in the $x$ - and $y$-direction and have the following terms:

$$
\begin{aligned}
& D_{x}^{-} u_{i, j, k}=\frac{u_{i, j, k}-u_{i, j-1, k}}{\Delta x}, \\
& D_{x}^{+} u_{i, j, k}=\frac{u_{i, j+1, k}-u_{i, j, k}}{\Delta x}, \\
& D_{y}^{-} u_{i, j, k}=\frac{u_{i, j, k}-u_{i, j, k-1}}{\Delta y}, \\
& D_{y}^{+} u_{i, j, k}=\frac{u_{i, j, k+1}-u_{i, j, k}}{\Delta y}, \\
&\left|D_{x}^{+} u_{i, j, k}\right|=\left(\left(\max \left(D_{x}^{-} u_{i, j, k}, 0\right)\right)^{2}+\left(\min \left(D_{x}^{+} u_{i, j, k}, 0\right)\right)^{2}\right. \\
&\left.+\left(\max \left(D_{y}^{-} u_{i, j, k}, 0\right)\right)^{2}+\left(\min \left(D_{y}^{+} u_{i, j, k}, 0\right)\right)^{2}\right)^{1 / 2} .
\end{aligned}
$$

The explicit discretization is given as:

$$
\begin{aligned}
& u_{i, j, k}^{n+1}=u_{i, j, k}^{n}+\Delta t F_{0}\left|D_{x}^{+} u_{i, j, k}^{n}\right|, \\
& \quad i=1, \ldots, M, j=1, \ldots, M_{x}, k=1, \ldots, M_{y}, n=0, \ldots, N, \\
& \quad u_{i, j, k}^{0}=u_{i, j, k, 0}, \text { initial condition, }
\end{aligned}
$$

where we have $F_{0} \geq 0$ based on the positive velocity $v_{x}, v_{y} \geq 0$ of the transport equations. Furthermore, $M$ is the number of bubbles, $M_{x}$ and $M_{y}$ are the number of spatial grid-points in $x$ and $y$ direction. $N$ is the number of time-steps.

For our case of the pure velocity in the $y$-directions, means we have $v_{x}=0, v_{y} \geq 0$, the discretization is simplified to:

$$
\begin{aligned}
& u_{i, j, k}^{n+1}=u_{i, j, k}^{n}+\Delta t F_{0}\left|\tilde{D}_{x}^{+} u_{i, j, k}^{n}\right|, \\
& \left|\tilde{D}_{x}^{+} u_{i, j, k}\right|=\left(\left(\max \left(D_{x}^{-} u_{i, j, k}, 0\right)\right)^{2}+\left(\min \left(D_{x}^{+} u_{i, j, k}, 0\right)\right)^{2}\right)^{1 / 2}, \\
& i=1, \ldots, M, j=1, \ldots, M_{x}, k=1, \ldots, M_{y}, n=0, \ldots, N, \\
& u_{i, j, k}^{0}=u_{i, j, k, 0}, \text { initial condition, }
\end{aligned}
$$

where $F_{0}=v_{y}$ is the pure velocity in $y$ direction.

An alternative approach of the shape transport can be done with the VOF method. This method is based on a free-surface modeling technique, while the method is tracking and locating the free surface, see also [17]. These methods are mass-conserved but more delicate to implement and more computationally intensive; therefore, we deal with the idea to reduce computational time with fast LS-methods and update the far-near-field coupling as much as possible to reduce the error of mass-conservation.

\section{Coupling Near-Field and Far-Field}

The modeling assumes that we could decouple the near- and far-field, while we neglect the coalescence or ruptures of the bubbles, such as in the flow-field, see [34].

We assume that in terms of the bubble density function:

$$
f_{b}(r, z, x, y, t)=u(x, y, t) \delta((r-R(x, y, t)),(z-Z(x, y, t))),
$$


where $u$ is the concentration of the bubble and $R$ and $Z$ are obtained with the bubble formation equations, while $r$ and $z$ are the cylinder coordinates of the density function, that we do not have an influence means $r \approx R$ and $z \approx Z$ for the formation process.

We discuss the following different coupling ideas:

- Decoupled computation of near- and far-field: parameters of the ellipse are computed in the near-field and initialize the far-field bubble.

- Coupled computation of near- and far-field: the near-field computation is directly implemented into the far-field by a so-called bubble-in-cell (BIC) method and is directly updated in a computational cycle.

\subsection{Decoupled Computation of Near-and Far-Field}

In the decoupled version, we only initialize the bubble and update the bubble shaped from time to time.

Here, we assume a nearly constant bubble, which is near the initialization of the bubble.

The bubble is computed by the near-field-model with the ODE's given in (5)-(7).

Then, we estimate the characteristic parameters of the ellipse in the Figure 7.

Estimation of the bubble-parameters

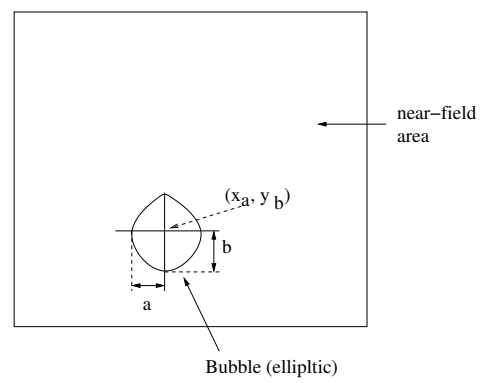

Figure 7. Final bubble based on the near-field computation and estimation of the bubble parameters (we assume an elliptic curve).

Based on the estimation of the elliptic-parameters, we obtain the curvature of the ellipse:

$$
\frac{\left(x-x_{a}\right)^{2}}{a^{2}}+\frac{\left(y-y_{b}\right)^{2}}{b^{2}}=1 .
$$

The ellipse is interpolated to the far-field model with shape-interpolations and we transport bubble shape with the LS-method.

The relatively stable bubble allows us to apply assumptions, such that we only update the bubble from time to time. The transformation of the elliptic parameters of the near-field model allows us to simplify the construction of the shape in the far-field. We only apply the ellipses in the far-field transport model. Therefore, we save computational time.

\subsection{Coupled Computation of Near-and Far-Field}

Based on the electric-field, which acts on a smaller time-scale, we have to update the near-field and far-field coupling in a numerical cycle,

The near-field bubble is computed with the ODE's and with the underlying influence of the E-field. Furthermore, the far-field is computed by the transport equation with level-set methods. We realize the coupling via an interpolation between the mesh-free space of the near-field and the grid-space of the far-field with the given diameters of the bubbles and the trajectories of the transport field

The numerical cycle is given in Figure 8. 


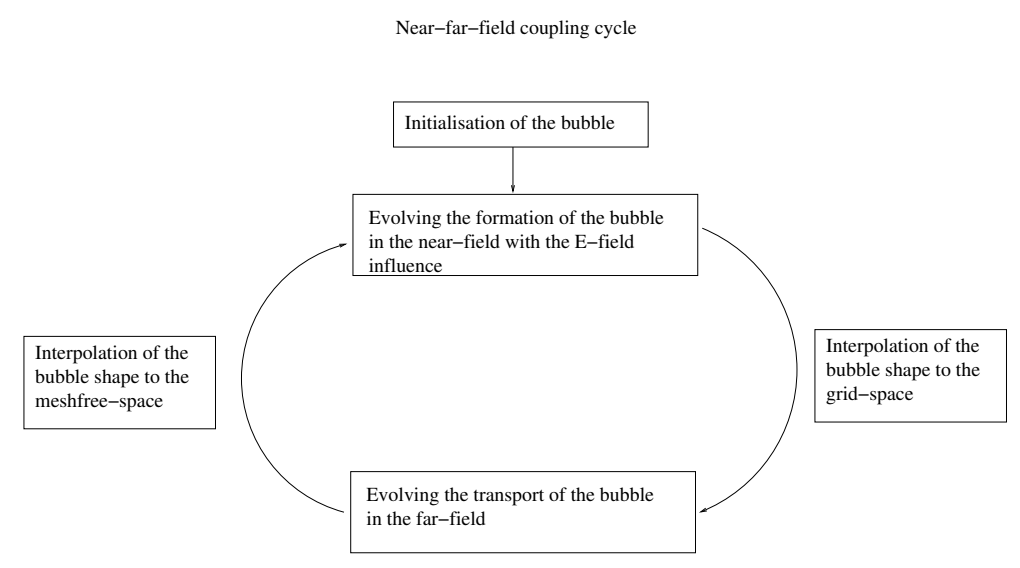

Figure 8. Numerical cycle of the near-far-field coupling.

In the following, we discuss the detailed coupling of the near-field and far-field, see Figure 9.

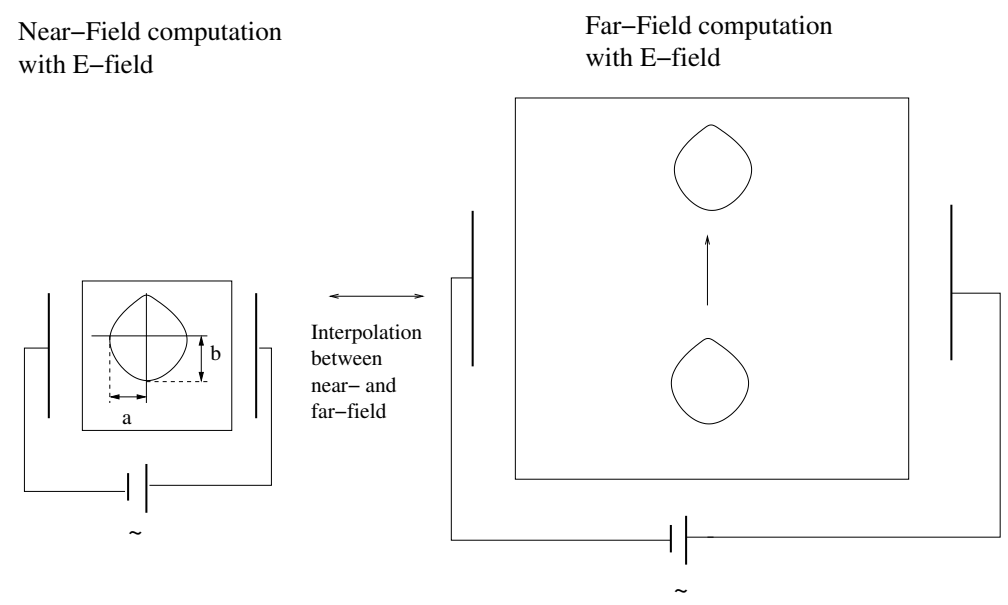

Figure 9. Near field and far field coupling in the E-field.

The accuracy of the cycle is given with respect to the step-size of the far-field. Further, we can improve the accuracy of the cycle with higher-order interpolation schemes and higher-order near-field and far-field solvers, see the improvement of the PIC-cycles [15,25]. Such ideas of different scale-dependent solvers can also be applied to so-called multiscale approaches, see [35].

A stronger coupling of the near- and far-field is given by an idea that is also used in the PIC (particle in cell) algorithms, see [14,15]. Here, we couple microscopic scales-that is, particle scales with macroscopic scales (i.e., clusters of particles in a macroscopic grid), see [36].

We apply a so called modified PIC algorithm, which we call the BIC (bubble in cell) algorithm. These algorithms use the Young-Laplace equation in the finer scales, while we apply the bubble formation and modification, and the level-set or transport equations in the coarser scales, while we apply the bubble transport, see [37].

The algorithm is given in the following Figure 10.

We have first order approximation errors for the interpolation between phase-space and grid space, while we apply a linear approximation, see [15]. We could improve the interpolation error with the help of higher order spline-approximations, such as second or third order splines, see [15]. This fine updating of the near- and far-field model allows to obtain more accurate results. 


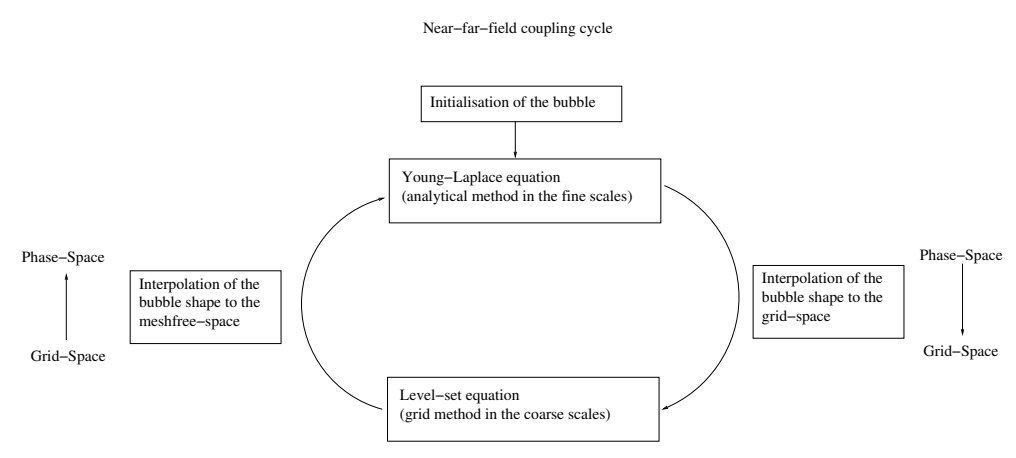

Figure 10. Bubble in cell algorithm based on the near-far-field approximation.

\section{Numerical Experiments}

In the following, we apply the different numerical experiments based on the bubble formation and the bubble transport. While the bubble formation is based on the model with Young-Laplace equation, the bubble transport model is based on the level-set equations.

\subsection{Bubble Formation: Experiment 1}

The near-field equations are given as:

$$
\begin{aligned}
& \frac{d r}{d s}=\cos (\theta), \\
& \frac{d z}{d s}=\sin (\theta), \\
& \frac{d \theta}{d s}=-\frac{\sin (\theta)}{r}+\frac{\Delta p}{\sigma},
\end{aligned}
$$

or in vectorial form:

$$
\frac{d \mathbf{y}}{d s}=\mathbf{f}(\mathbf{y}, s)=\tilde{A}(\mathbf{y}(s))+\tilde{\mathbf{q}}(s), s \in\left[s_{i}, s_{i+1}\right],
$$

where $\tilde{A}(\mathbf{y}(s))=\left(\begin{array}{c}\cos (\theta) \\ \sin (\theta) \\ -\frac{\sin (\theta)}{r}\end{array}\right)$ and $\tilde{\mathbf{q}}(s)=\left(\begin{array}{c}0 \\ 0 \\ \frac{\Delta p}{\sigma}\end{array}\right)$. Furthermore, $\mathbf{y}(s)=\left(\begin{array}{c}r(s) \\ z(s) \\ \theta(s)\end{array}\right)$ and $s$ is the arc length along the curve and $r$ is the radius, $z$ is the vertical distance and $\theta$ the angle of elevation for its slope and $\sigma$ is the mono-layer surface tension.

We have to linearize the equations, which are given as:

$$
\begin{aligned}
& \frac{d \mathbf{y}}{d t}=\tilde{A}\left(\mathbf{y}\left(s_{i}\right)\right)+\left.\frac{\partial \tilde{A}}{\partial \mathbf{y}}\right|_{\mathbf{y}\left(s_{i}\right)}\left(\mathbf{y}(s)-\mathbf{y}\left(s_{i}\right)\right)+\tilde{\mathbf{q}}(s), s \in\left[s_{i}, s_{i+1}\right], \\
& \frac{d \mathbf{y}}{d s}=A(s) \mathbf{y}(s)+\mathbf{q}(s), t \in\left[s_{i}, s_{i+1}\right], \\
& B_{a} \mathbf{y}(a)+B_{b} \mathbf{y}(b)=\mathbf{d},
\end{aligned}
$$

where $A(t)=\left.\frac{\partial \tilde{A}}{\partial \mathbf{y}}\right|_{\mathbf{y}\left(s_{i}\right)}, \mathbf{q}(s)=\tilde{A}\left(\mathbf{y}\left(s_{i}\right)\right)-\left.\frac{\partial \tilde{A}}{\partial \mathbf{y}}\right|_{\mathbf{y}\left(s_{i}\right)} \mathbf{y}\left(s_{i}\right)+\tilde{\mathbf{q}}(s)$. In the linearization, we apply the Jacobian matrix, which is given as

$$
\left.\frac{\partial A}{\partial \mathbf{y}}\right|_{\mathbf{y}\left(s_{i}\right)}=\left(\begin{array}{ccc}
0 & 0 & -\sin \left(\theta\left(s_{i}\right)\right) \\
0 & 0 & \cos \left(\theta\left(s_{i}\right)\right) \\
-\frac{\sin \left(\theta\left(s_{i}\right)\right)}{r^{2}\left(s_{i}\right)} & 0 & -\frac{\cos \left(\theta\left(s_{i}\right)\right)}{r\left(s_{i}\right)}
\end{array}\right)
$$


We have the conditions:

$$
\begin{aligned}
& r=a, z=0, \theta=\pi / 2, \text { at } s=0, \\
& r=0, \frac{d z}{d s}=0, \theta=2(\pi / 2), \text { at } s=L,
\end{aligned}
$$

where $a$ is the radius of the bubble, $L$ is the arc length of the bubble which is a-priori unknown, where we start with $L=L_{0}=\frac{2 \pi a^{2}}{4}$ and we go on with $L=L_{1} \leq L_{2} \leq \ldots \leq L_{\text {stop }}$.

We have the following linear equation system:

$$
\left[\begin{array}{lllll}
S_{1} & R_{1} & & & \\
& S_{2} & R_{2} & & \\
& & \ddots & \ddots & \\
& & & S_{N} & R_{N} \\
B_{a} & & & B_{b-1} & B_{b}
\end{array}\right]\left[\begin{array}{l}
y_{1} \\
y_{2} \\
\vdots \\
y_{N} \\
y_{N+1}
\end{array}\right]=\left[\begin{array}{l}
q_{1} \\
q_{2} \\
\vdots \\
q_{N} \\
d
\end{array}\right],
$$

where $S_{i}=R_{i}=-h_{i}^{-1} I-\frac{1}{2} A\left(s_{i+1 / 2}\right), q_{i}=q\left(s_{i+1 / 2}\right.$ with $h_{i}=s_{i+1}-s_{i}, s_{i+1 / 2}=s_{i}+\frac{1}{2} h_{i}$.

Furthermore, we have matrices and vectors:

$$
B_{a}=\left(\begin{array}{ccc}
0 & 0 & 0 \\
0 & 0 & 0 \\
0 & 0 & 0
\end{array}\right), B_{b-1}=\left(\begin{array}{ccc}
0 & 0 & 0 \\
0 & 1 & 0 \\
0 & 0 & 0
\end{array}\right), B_{b}=\left(\begin{array}{lll}
1 & 0 & 0 \\
0 & 1 & 0 \\
0 & 0 & 1
\end{array}\right), \mathbf{d}=\left(\begin{array}{c}
L \\
0 \\
0
\end{array}\right) .
$$

- $\quad$ Test example 1:

We apply the following test-example with the a quarter of a circle, means $L=2 \pi a^{2} / 4$, where $a=1,2,3,3.5$ and we assume $N=L / \Delta s$, where we assume $\Delta s=2 \pi \cdot 0.001$ is the arc-length step for the numerical computations. Further, we have $\frac{\Delta p}{\sigma}=0$.

- $\quad$ Test example 2:

We have $N=L / \Delta s$, where we assume $\Delta s=0.001$ is the arc-length step for the numerical computations. Further, we have $L=2$ and $\frac{\Delta p}{\sigma}=0,0.8,1.4$.

The numerical results of bubble formation are given in Figure 11.
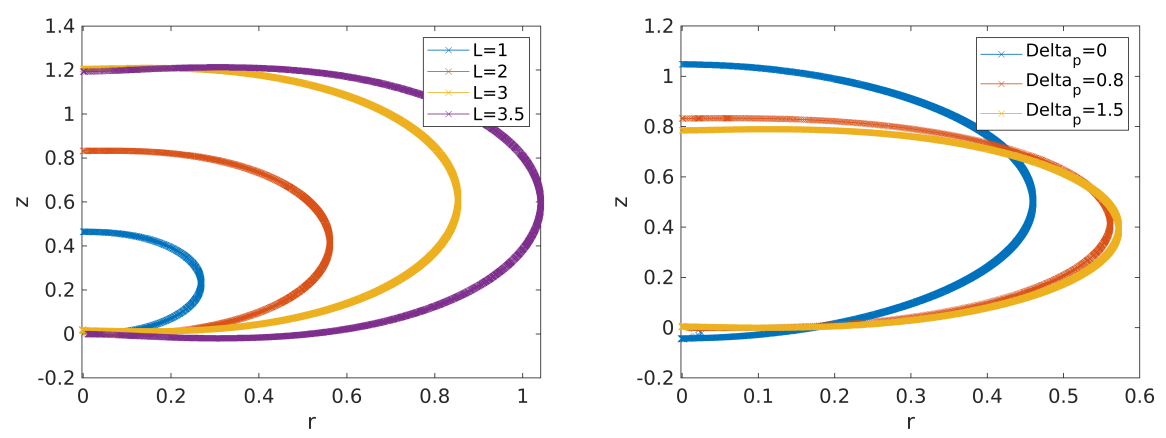

Figure 11. Bubble formation, left figure with different lengths $L$ and $\frac{\Delta p}{\sigma}=0$, while $L=2$ is exact solution of the $1 / 4$ circle and right-hand figure with different right-hand side-parameters $\frac{\Delta p}{\sigma}$ and for $L=2$.

The Young-Laplace equation allows us to formulate the bubble formation, such that we could obtain the radii of the different bubbles based on the various pressure parameters. We also compare the results with respect to the computations in the literature, see the ideas in [16]. 


\subsection{Bubble Formation: Validation of the Near-Field}

In the following, we validate the near-field model, which is a model based on the formation of bubbles.

We can obtain an analytical expression, see [29]. Based on the mechanical equilibrium approach, we can develop the analytical expressions and predict the bubble departure volume. This departure volume is based on the force balance analysis under different assumptions, see also [38].

The relationship is given as:

$$
V=N \frac{Q^{6 / 5}}{g^{3 / 5}},
$$

$g$ is the gravity, $N$ is a constant with $N=1.378$. The departure volume is given as $V$ and the gas-flow rate is $Q$.

We apply different experiment to validate with the analytical expression:

- $\quad$ Test example 1:

We apply the following test-example with the circumference of the ellipse, which are given as $L_{i}=2 \pi a_{i}^{2} / 4$, where $L_{1}=1, L_{2}=2, L_{3}=3, L_{4}=3.5, L_{5}=4.0$, while a special case is the circle with $L_{\text {circ }}=\pi / 2$ and radius $r=1$. Furthermore, we assume $N_{i}=L_{i} / \Delta s$, where the arc-length step for the computations is $\Delta s=2 \pi \cdot 0.001$ and $\frac{\Delta p}{\sigma}=0$.

Here, we measure prediction of the volume of the ellipsoid, while we assume a constant gas-flow-rate:

$$
Q_{L_{i}}=\frac{d V_{L_{i}}}{d t}
$$

means we apply:

$$
Q_{L_{i}}=\frac{V_{L_{i}}\left(t_{2}\right)-V_{L_{i}}\left(t_{1}\right)}{t_{2}-t_{1}}
$$

where $i=1,2,3,4$, which we could compute with our MATLAB simulations. We assume that $Q$ is a constant and approximated to $V$.

Then, we can predict the departure volume with the experimental formula:

$$
V_{L_{i}}=N \frac{Q_{L_{i}}^{6 / 5}}{g^{3 / 5}}
$$

where the constants are defined in Equation (58) and different volumes of the ellipsoids are given with the indices $i=1,2,3,4$.

In the following Figure 12, we present the validation of the numerical computed volumes $V_{n u m, L_{i}} / V_{n u m, L_{i-1}}$ and the experimental computed volumes $V_{L_{i}} / V_{L_{i-1}}$. The experimental computed volumes are given with the Equation (61), see the experiments in [29]. 


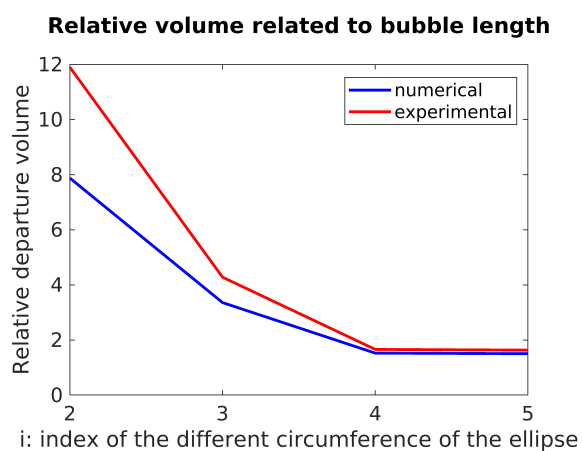

Figure 12. Validation for the relative departure volume related to the bubble length $L_{i}$, which are based on the numerical volume $V_{n u m, L_{i}}$ and the experimental volume $V_{L_{i}}$.

- $\quad$ Test example 2:

We have $N=L / \Delta s$, where the arc-length step for the computations is $\Delta s=0.001$ and $L=2$ and we apply the following pressure relations $\frac{\Delta p_{1}}{\sigma}=0, \frac{\Delta p_{2}}{\sigma}=0.5, \frac{\Delta p_{3}}{\sigma}=1.0, \frac{\Delta p_{4}}{\sigma}=1.5$ and $\frac{\Delta p_{5}}{\sigma}=2.0$. Here, we can predict the different gas-flow-rates, based on the different pressure relations.

$$
Q_{\Delta p_{i} / \sigma}=\frac{V_{\Delta p_{i} / \sigma}\left(t_{2}\right)-V_{\Delta p_{i} / \sigma}\left(t_{1}\right)}{t_{2}-t_{1}}
$$

where $i=1,2,3,4,5$, which we could compute with our MATLAB simulations and we assume $Q$ is a constant and approximated to $V$.

Then, we can predict the departure volume with the experimental formula:

$$
V_{\Delta p_{i} / \sigma}=N \frac{Q_{\Delta p_{i} / \sigma}^{6 / 5}}{g^{3 / 5}}
$$

where the constants are defined in Equation (58) and different pressure relations are given with the indices $i=1,2,3,4$.

In the following Figure 13, we present the validation of the numerical computed volumes $V_{\text {num }, \Delta p_{i} / \sigma} / V_{n u m, \Delta p_{i-1} / \sigma}$ and the experimental computed volumes $V_{\Delta p_{i} / \sigma} / V_{\Delta p_{i-1} / \sigma}$. The experimental computed volumes are given with the Equation (63), see the experiments in [29].

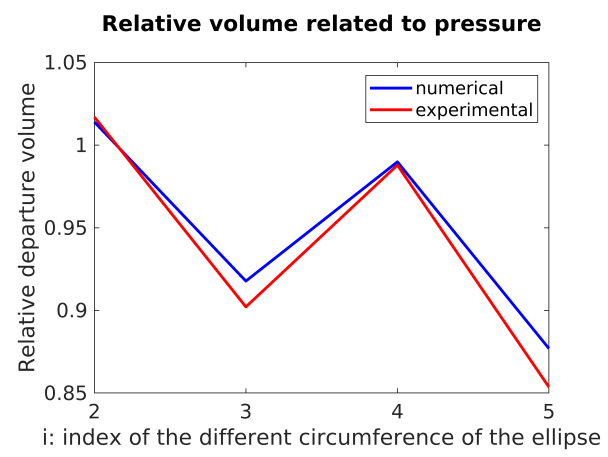

Figure 13. Validation for the relative departure volume related to the bubble length $L_{i}$, which are based on the numerical volume $V_{n u m, L_{i}}$ and the experimental volume $V_{L_{i}}$.

We have applied the validation of the near-field model simulation with the experimentally results of the heuristic formulas (61) and (63). We compared the experimental and numerical results and 
obtain relatively the same results. Such a validation allows us to apply the near-field model for the simulations of the far-field model, see also bubble-experiments in [39].

\subsection{Bubble Transport: Validation of the Far-Field}

In the following, we validate the far-field model, which is a model based on the transport of bubbles.

We define $N$ grid points for the $x$ and $y$ direction in the area of the bubble transport. Then, we deal with different space-steps, such as $\Delta x, \Delta x / 2, \Delta x / 4, \Delta x / 8$.

We apply the numerical error based on the $L_{2}$-norm:

$$
\operatorname{err}_{L_{2}, \Delta x, \Delta x / 2}(t)=\frac{1}{N} \sum_{i=1}^{N}\left(u_{i, \Delta x}(t)-u_{i, \Delta x / 2}(t)\right)^{2},
$$

where $N$ number of grid-points on which we compute the solutions. $\Delta x$ spatial step of the error analysis. $t$ time, where we apply the convergence study, e.g., $t=t_{\text {end }}$.

Furthermore, we apply the experimental order of convergence, see [40], which is given as:

$$
\rho_{L_{2}, \Delta x, \Delta x / 2}(t)=\frac{\log \left(\frac{\operatorname{err}_{L_{2}, \Delta x / 2, \Delta x / 4}(t)}{\operatorname{err}_{L_{2}, \Delta x, \Delta x / 2}(t)}\right)}{\log (0.5)}
$$

where we can see a convergence rate based on the refinement of the spatial grid.

We have the following Table 1 for the validation of the far-field:

Table 1. Validation for the far-field solvers with $N=N_{x}=N_{y}=41$.

\begin{tabular}{ccc}
\hline$\Delta x$ & err $_{L_{2}, \Delta x, \Delta x / 2}(t)$ & $\rho_{L_{2}, \Delta x, \Delta x / 2}(t)$ \\
\hline $1 / N$ & $1.210^{8}$ & \\
$1 /(2 N)$ & $7.810^{7}$ & 0.621 \\
$1 /(4 N)$ & $2.110^{7}$ & 1.893 \\
$1 /(8 N)$ & $3.210^{6}$ & 2.714 \\
\hline
\end{tabular}

In the following Figure 14, we see the convergence rates with the higher resolution of the computations.

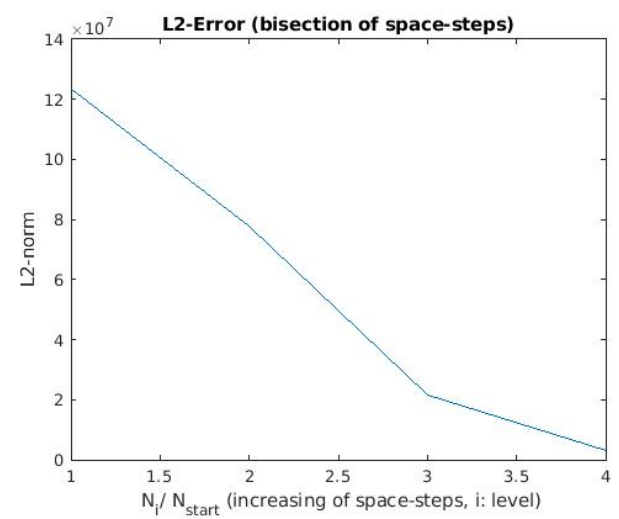

Figure 14. Convergence analysis of the finer resolution with respect to the far-field validation with $N_{\text {start }}=41$ and $N_{i}=i N_{\text {start }}$.

We see a convergence based on the $L_{2}$-error with finer resolutions in Figure 14 . Therefore, we obtain with finer grids a higher resolution for the far-field computations. Such a convergence analysis allows us to validate the far-field computations. 


\subsection{Bubble Formation: Experiment 2}

In the following, we couple the near-field and far-field computations.

We have the following setting, see Figure 15.

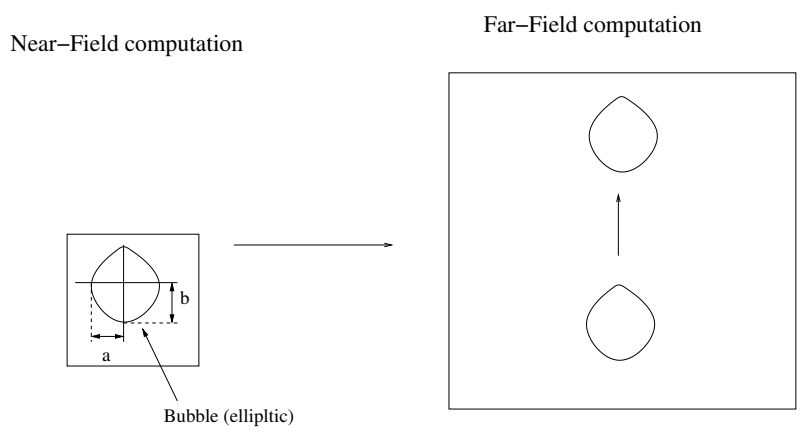

Figure 15. (left) Bubble formation with the ordinary differential equations (ODEs). (right) Bubble transport with the partial differential equations (PDEs) (level-set equations).

We apply the following parameters:

- Input-parameters of the near-field bubbles:

- $r_{1}(0)=0.01, z_{1}(0)=0, r_{1}(L)=0, L=1, \frac{\Delta p_{1}}{\sigma_{1}}=0.8$,

- $r_{2}(0)=0.01, z_{2}(0)=0, r_{2}(L)=0, L=2, \frac{\Delta p_{2}}{\sigma_{2}}=0.8$.

- We compute the bubbles based on the near-field code and we obtain the ellipse-diameters $a_{\text {bubble, }} b_{\text {bubble }}$.

- We initialize the two ellipses for the far-field computations given as:

- $\quad(x-20)^{2}+\left((y-100) * a_{\text {bubble, } 1} / b_{\text {bubble, } 1}\right)^{2}-a_{\text {bubble, } 1}^{2}$

$-(x-70)^{2}+\left((y-90) * a_{\text {bubble,2 }} / b_{\text {bubble }, 2}\right)^{2}-a_{\text {bubble, } 2}^{2}$.

The numerical results of near-field bubble formation are given in Figure 16.

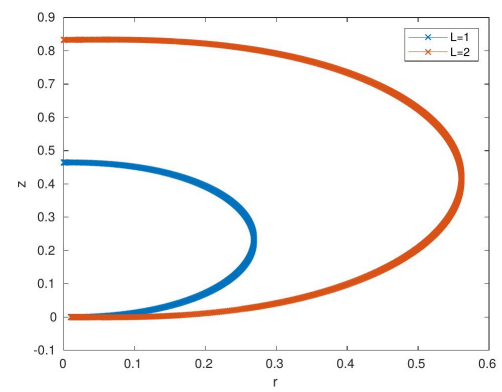

Figure 16. The computation of the bubble formation (near-field) for two bubbles.

The numerical results of the near-far-field coupled bubble transport code, which is given in Figure 17.

The coupling of the formation and transport of the bubbles is done with ordinary and partial differential equations. Such a coupling closes the gap between the different scale-dependent models of a near-field and far-field simulation, see [37]. Based on decoupling such systems of mixed ordinary and partial differential equations, we were able to compute each separate part with the optimal numerical solvers. We apply higher order near-field and far-field solvers, which accelerate the computational time, see also [25,37]. 

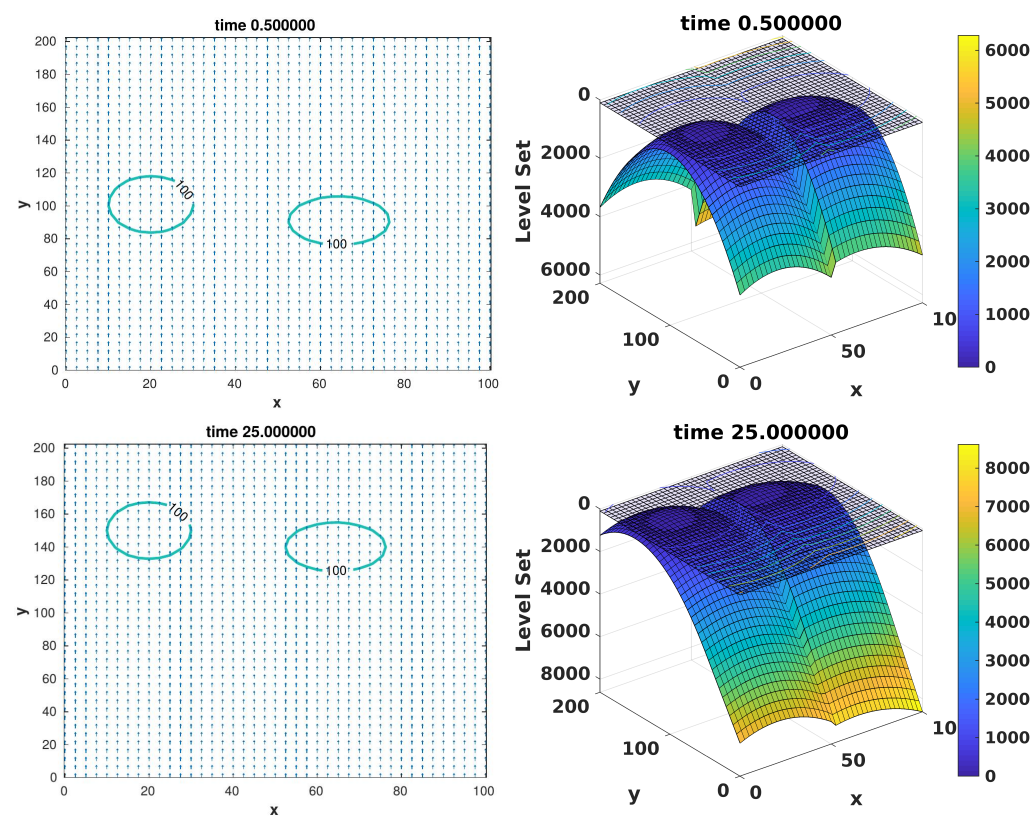

Figure 17. (top left) Transport of the two bubbles in the far-field at time $t=0.5$. (top right) Level-set function of the two bubbles at time $t=0.5$ with the level-set-area of 100 . (bottom left) Transport of the two bubbles in the far-field at time $t=25.0$. (bottom right) Level-set function of the two bubbles at time $t=25.0$ with the level-set-area of 100 .

\subsection{Bubble Formation: Multiple Bubble Experiment (10 Bubbles)}

In the following, we extend the near-field and far-field computations with 10 bubbles. We also apply the decomposition of near-field and far-field computations as given in Figure 15.

We apply the following parameters:

- Computation of the near-field bubbles (a representing bubble is computed):

- Input-parameters of the near-field bubbles computation are given in Appendix A.1.

- Output-parameters of the near-field bubble computation are given in Appendix A.1.

- Ellipse: $\left(x-x_{\text {bubble }_{i}}\right)^{2}+\left(\left(y-y_{\text {bubble }_{i}}\right) * a_{\text {bubble }} / b_{\text {bubble }}\right)^{2}-a_{\text {bubble }}^{2}$ where $\left(x_{\text {bubble }_{i}}, y_{\text {bubble }_{i}}\right)$ is the origin of the $i$-th bubble.

- Computation of the far-field bubbles (level-set initialization):

- Parameterization of the level-set initial-function, such as two bubbles:

$$
\phi_{0}(x, y)=\left\{\begin{array}{c}
\left(x-x_{\text {bubble }_{1}}\right)^{2}+\left(\left(y-y_{\text {bubble }_{1}}\right) \frac{a_{\text {bubble }_{1}}}{b_{\text {bubble }_{1}}}\right)^{2}-a_{\text {bubble }_{1}}^{2} \\
a_{x} \leq x \leq 50, a_{y} \leq x \leq b_{y} \\
\left(x-x_{\text {bubble }_{2}}\right)^{2}+\left(\left(y-y_{\text {bubble }_{2}}\right) \frac{a_{\text {bubble }}}{b_{\text {bubble }_{2}}}\right)^{2}-a_{\text {bubble }_{2}}^{2} \\
50 \leq x \leq b_{x}, a_{y} \leq x \leq b_{y}
\end{array}\right.
$$

where $\left(x_{\text {bubble }_{1}}, y_{\text {bubble }_{1}}\right)=(20,50),\left(x_{\text {bubble }_{2}}, y_{\text {bubble }_{2}}\right)=(80,50)$ with the coordinates of the $\operatorname{grid}\left(a_{x}, a_{y}\right)=(0,0)$ and $\left.b_{x}, b_{y}\right)=(100,200)$.

The numerical results of the formation of the bubbles are given in Figure 18. 


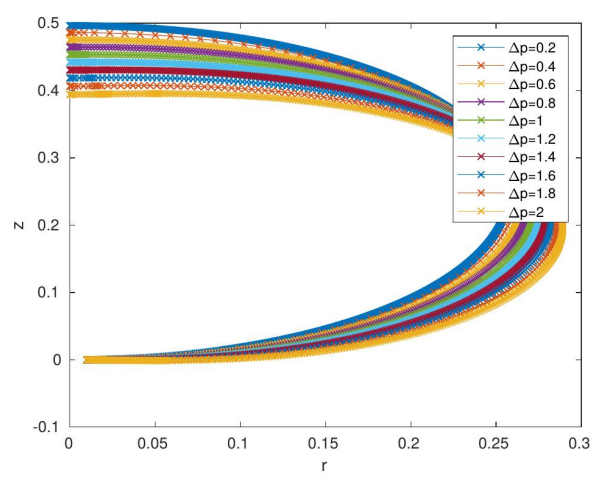

Figure 18. Formation of 10 bubbles with the different pressure-terms.

The numerical results of the near-far-field coupled bubble transport code, which is given in Figures 19 and 20.
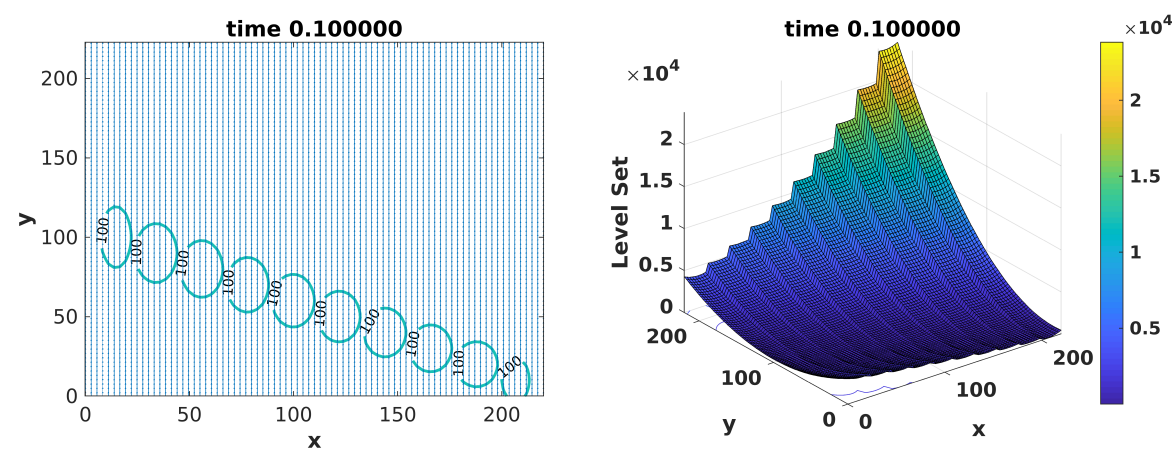

Figure 19. Transport of 10 bubbles with the level-set function at the initialization $t=0.1$.
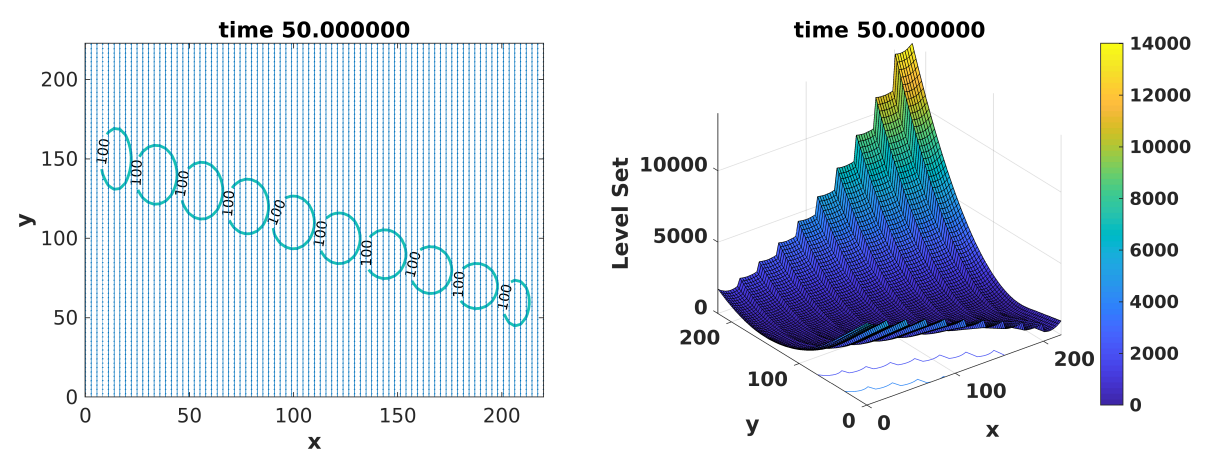

Figure 20. Transport of 10 bubbles with the level-set function at time $t=50$.

In the experiment, we deal with at least 10 bubbles, which are differently formatted and transported via the level-set method. Such experiments are important to simulate experiments, which are used for bubble reactors, see [2,6]. The numerical experiments allow us to accelerate the formation and transport of such processes and save expensive laboratory experiments, see [41].

\subsection{Bubble Formation: Oscillation of the Air-Bubbles in the Electrical-Field (Decoupled Version)}

In the following, we simulate a bubble filled with air in an electrical field, see [10]. We apply the decoupled computation of the near- and far-field, as discussed in Section 5.1.

We have the following setting of the influenced bubble, see Figure 21. 
Bubble influenced in an E-field

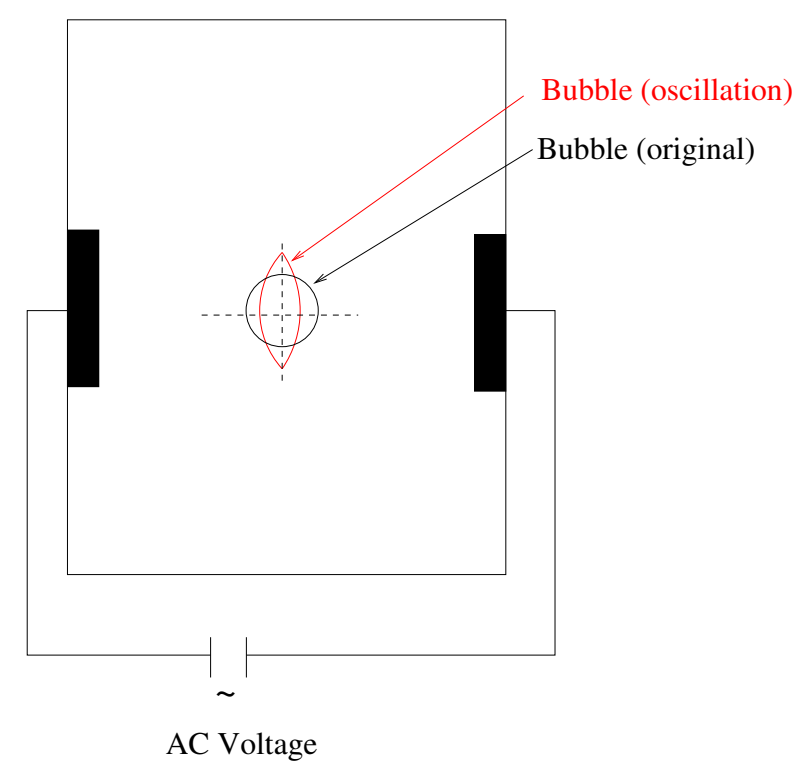

Figure 21. Bubble formation influenced by an electrical field.

We apply the following near-field equation based on an extension of the pressure-term with an E-field.

The near-field equations with the E-field are given as:

$$
\begin{aligned}
& \frac{d r}{d s}=\cos (\theta), \\
& \frac{d z}{d s}=\sin (\theta), \\
& \frac{d \theta}{d s}+\frac{\sin (\theta)}{r}=\frac{\Delta p}{\sigma}+\frac{p_{E}}{\sigma},
\end{aligned}
$$

where $s$ is the arc length along the curve and $\theta$ the angle of elevation for its slope and $\sigma$ is the mono-layer surface tension, and the approximated pressure difference is given as $\Delta p=\frac{2}{R_{0}}$, see Section 2.1. $p_{E}=\frac{9}{8}\left|\mathbf{E}_{0}\right|^{2} \sin (\theta)$ is the electrical stress related to an external electrical field $\mathbf{E}_{0}$.

We apply the following parameters:

- Computation of the near-field bubbles (a representing bubble is computed)

- The electrical field parameters are given as:

$\sigma=0.1, \rho=0.1, g=9.81,\left|\mathbf{E}_{0}\right|^{2}=0.1$.

- Input-parameters of the near-field bubbles computation are given in Appendix A.2.

- Output-parameters of the near-field bubble computation (formation) are given in the Appendix A.2.

- Output-parameters of the near-field bubble computation (in the E-field) are given in Appendix A.2.

- Ellipse: $\left(x-x_{\text {bubble }_{i}}\right)^{2}+\left(\left(y-y_{\text {bubble }}\right) * a_{\text {bubble }} / b_{\text {bubble }}\right)^{2}-a_{\text {bubble }}^{2}$ where $\left(x_{\text {bubble }_{i}}, y_{\text {bubble }_{i}}\right)$ is the origin of the $i$-th bubble.

- Computation of the far-field bubbles (level-set initialization): 
- Parameterization of the level-set initial-function, such as two bubbles:

$$
\phi_{0}(x, y)=\left\{\begin{array}{c}
\left(x-x_{\text {bubble }_{1}}\right)^{2}+\left(\left(y-y_{\text {bubble }_{1}}\right) \frac{a_{\text {bubble }_{1}}}{b_{\text {bubble }_{1}}}\right)^{2}-a_{\text {bubble }_{1}}^{2} \\
a_{x} \leq x \leq 50, a_{y} \leq x \leq b_{y} \\
\left(x-x_{\text {bubble }_{2}}\right)^{2}+\left(\left(y-y_{\text {bubble }_{2}}\right) \frac{a_{\text {bubble }_{2}}}{b_{\text {bubble }_{2}}}\right)^{2}-a_{\text {bubble }_{2}}^{2} \\
50 \leq x \leq b_{x}, a_{y} \leq x \leq b_{y}
\end{array}\right.
$$

where $\left(x_{\text {bubble }_{1}}, y_{\text {bubble }_{1}}\right)=(20,50),\left(x_{\text {bubble }_{2}}, y_{\text {bubble }_{2}}\right)=(80,50)$ with the coordinates of the $\operatorname{grid}\left(a_{x}, a_{y}\right)=(0,0)$ and $\left.b_{x}, b_{y}\right)=(100,200)$.

The numerical results of the formation of the bubbles with and without the E-field are given in Figure 22.
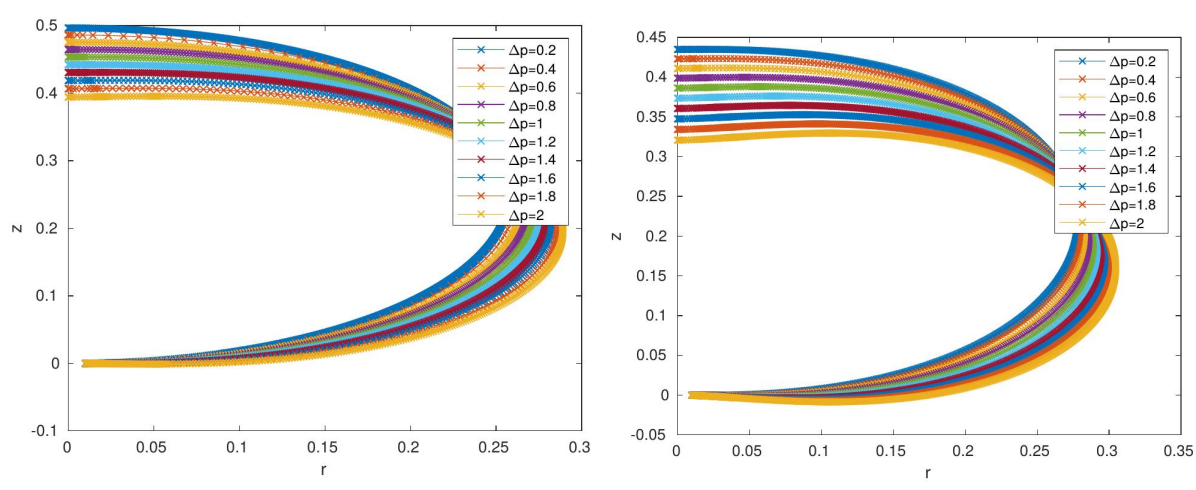

Figure 22. (left) Formation of 10 bubbles without an E-field. (right) Formation of 10 bubbles with an E-field.

The numerical results of the near-far-field coupled bubble transport code are given in Figures 23 and 24 .

The bubble modifications are given by the E-field, while it changes the formation of the bubble, see the Figure 24. The level-set method is flexible and is modified by multi-level-set domains. Therefore, it allows us to deal with multiple level-set functions of different concentrations and initial conditions, such that we could transport multiple bubbles with E-fields. Such a flexibilization is necessary to deal with the laboratory experiments, see [29].
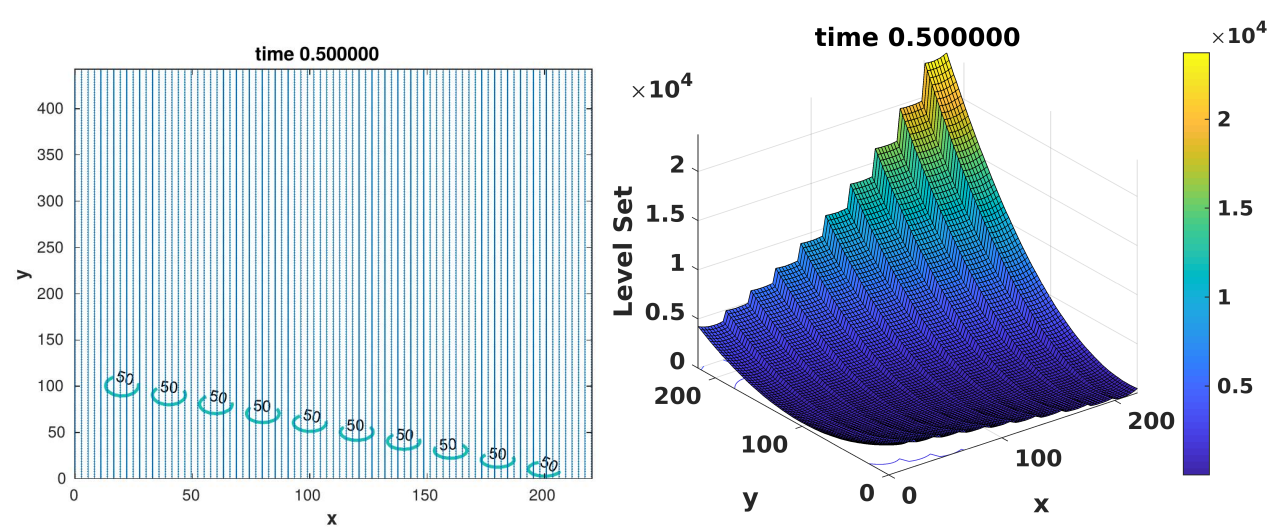

Figure 23. Transport of 10 bubbles in the E-field with the level-set function at the initialization $t=0.5$. 

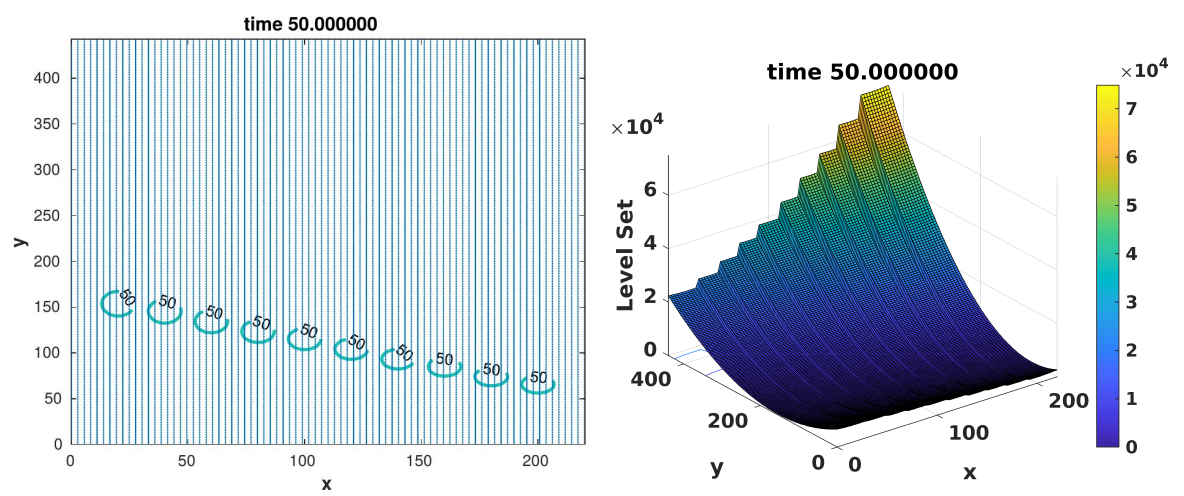

Figure 24. Transport of 10 bubbles in the E-field with the level-set function at time $t=50$.

\subsection{Bubble Formation: Oscillation of the Air-Bubbles of an Oscillating Electrical- Field (Coupled Version)}

In the following, we extend the near-field and far-field computations with additional coupling with an E-field, while we apply the cyclic computation of near- and far-field, see Figure 9. We apply the coupled computation of the near- and far-field, as discussed in Section 5.2.

The oscillation E-field is given in Figure 25, with the function:

$$
\begin{aligned}
& \left|\mathbf{E}_{0}\right|^{2}=0.1 f(t), \\
& f(t)=\left\{\begin{array}{ll}
1, & (n-1) 10 \leq t<n 10, \\
0, & n 10 \leq t<(n+1) 10,
\end{array}, \text { where } n=1,3,5, \ldots\right.
\end{aligned}
$$

\section{Oscillating E-field}

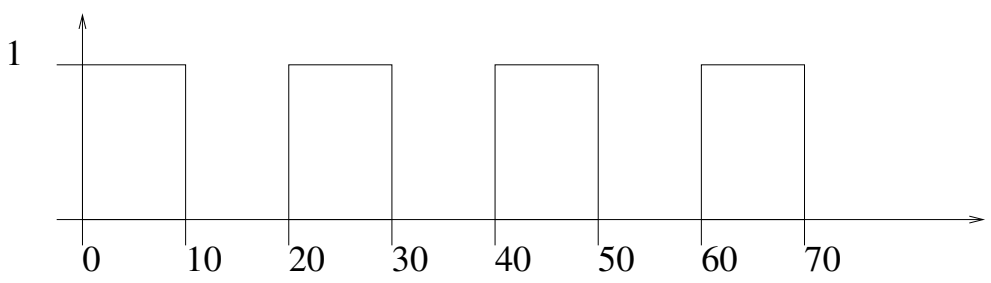

Figure 25. Oscillating E-field for the bubble formation.

We apply the same parameters as for the experiment of Section 6.6.

We apply the following algorithm, given in Figure 26 for computing the coupled E-field with the bubble transport and formation.

We applied the update of the E-fields, which means that we computed several transport steps in a switched-on the E-field, and applied several transport steps to a switched-off the E-field. While we have a faster transport-scale, it is sufficient to update the E-field from time to time.

The numerical results of the oscillating bubbles are given in the Figures 27 and 28.

In the experiment, we deal with at least 10 bubbles, which are differently formatted and transported via the multi-level-set method, see [13]. We apply an oscillating E-field and reinitialize the level-set method with the new formatted bubbles for each 5-10 time-steps. As a result, we see the oscillating bubbles in the E-field, which is important so apply the plasma-ignition, see [23]. The numerical experiments allow us to accelerate the formation and transport of such processes with a reduction of updates of the E-field, such that we could redo costly laboratory experiments. 


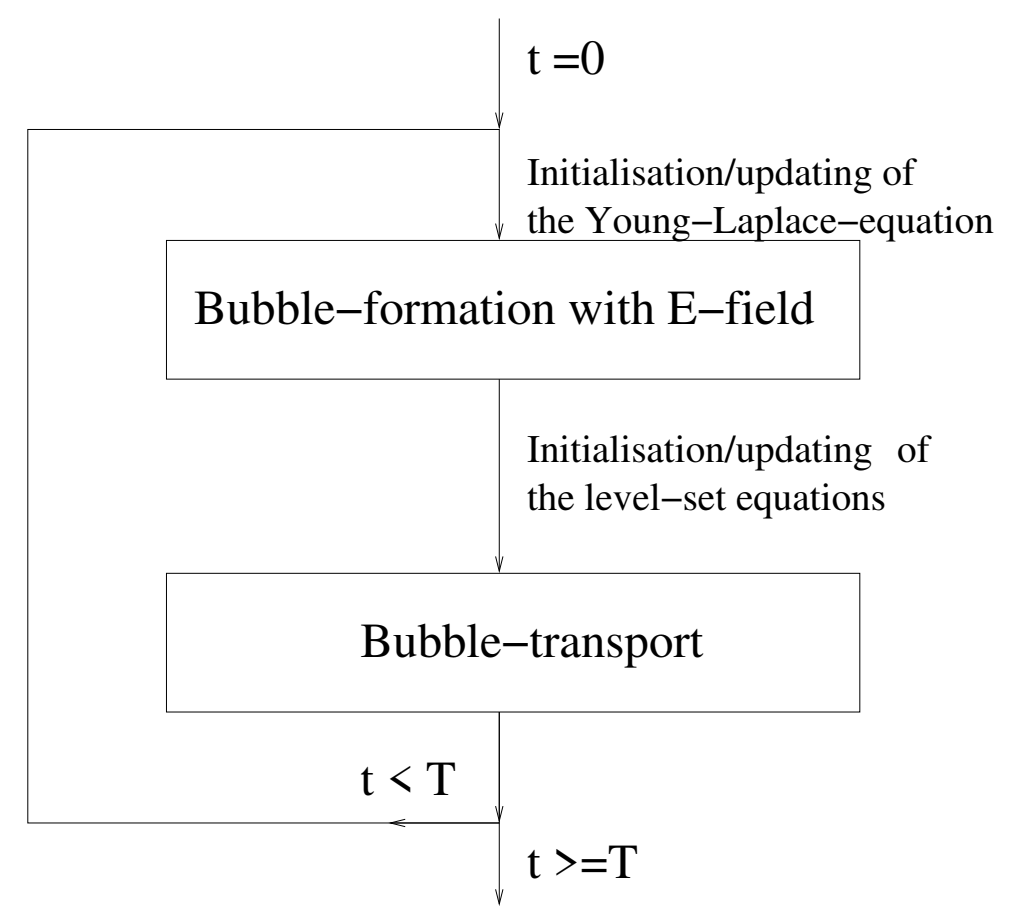

Figure 26. Algorithm for updating a bubble formation with an oscillating E-field in a transport regime based on level-set methods.
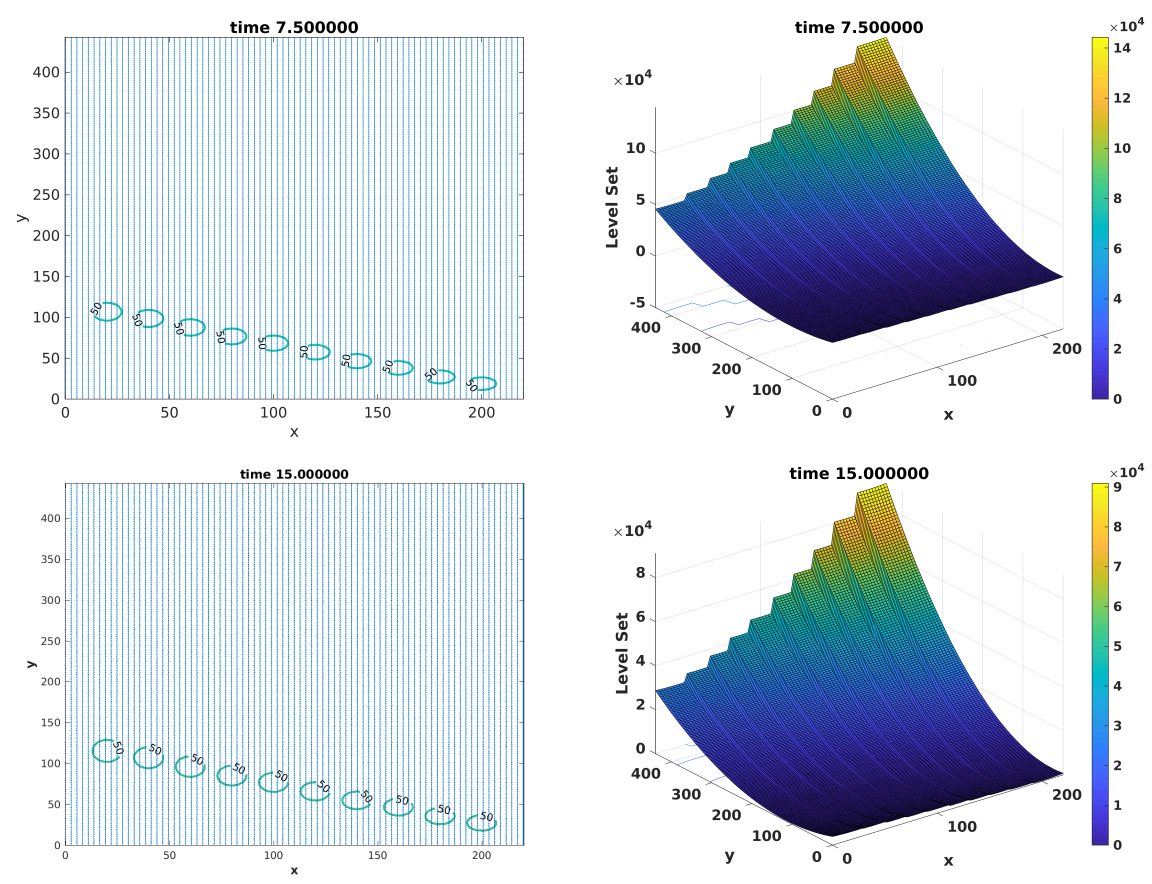

Figure 27. Transport of 10 bubbles with the level-set function: (top) $t=7.5$ with E-field switched off and (bottom) $t=15.5$ with E-field switched on. 

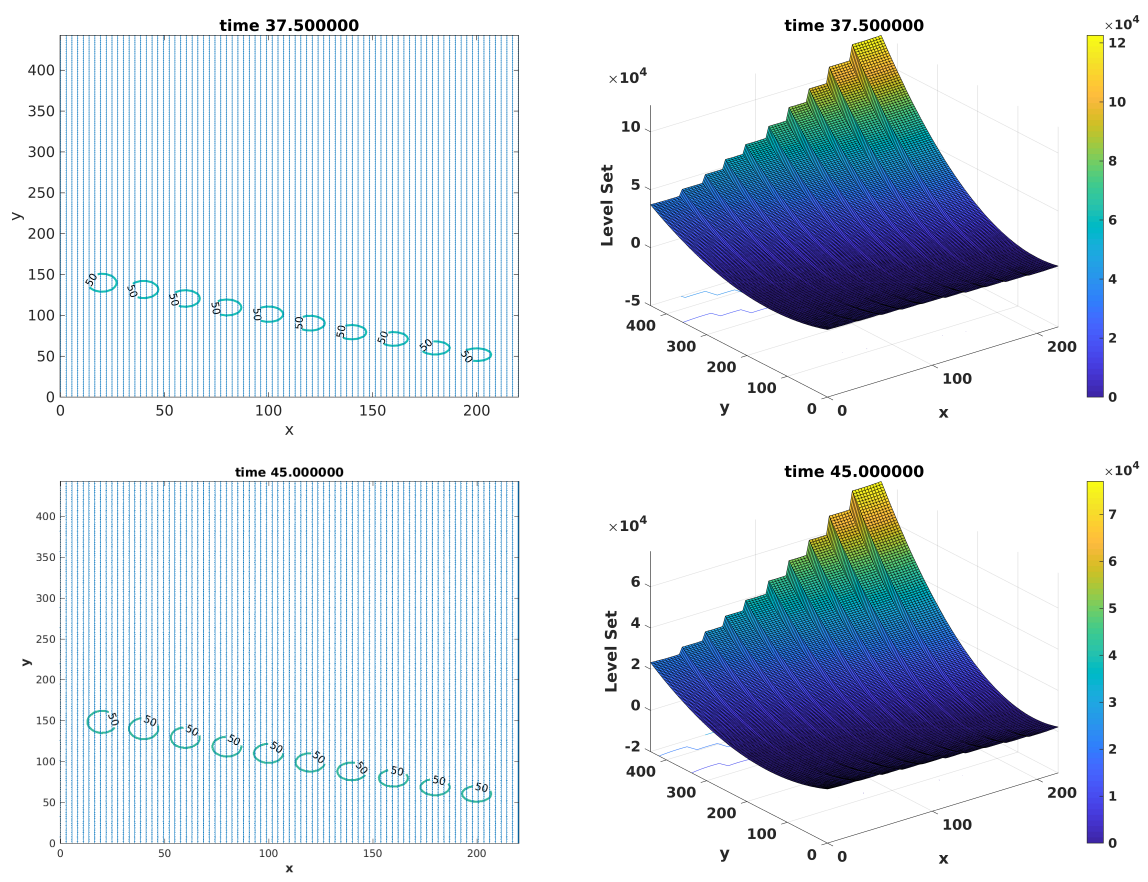

Figure 28. Transport of 10 bubbles with the level-set function: (top) at $t=38.0$ with E-field switched off and (bottom) $t=45.5$ with E-field switched on.

\section{Conclusions}

We present a bubble model, which is a coupled model based on a bubble formation and bubble transport model. The decoupling into near- and far-field models allows us to apply optimal solver and discretization methods. Such modeling allows us to close the gap of coupling scale-dependent transport-problems. While we could reduce the computational time in the near- and far-field model with the scale-optimized methods, we partly replace costly laboratory experiments. We apply different numerical experiments of the near-field and far-field model and presented their benefit in the simulations. Further, we present the oscillations of the charged bubbles in an electrical field, such results are important for possible plasma-ignitions. In the future, we will consider the fully coupled problem, while we deal with bubble density functions and the coupling between the formation and transport process. This extension allows us to see the ruptures of the bubbles.

Author Contributions: The theory, the formal analysis and the methology presented in this paper was developped by J.G. The software development and the numerical validation of the methods was done by P.M. and with the help of J.G. The paper was written by J.G. and was corrected and edited by J.G. and P.M. The writing-review was done by J.G. The supervision and project administration was done by J.G.

Funding: This research was funded by German Academic Exchange Service grant number 91588469.

Acknowledgments: We acknowledge support by the DFG Open Access Publication Funds of the Ruhr-Universität of Bochum, Germany.

Conflicts of Interest: The authors declare no conflict of interest.

\section{Appendix A.}

In the following, we give the parameters of the experiments in Sections 6.5-6.7.

\section{Appendix A.1. Parameters of Section 6.5}

- Input-parameters of the near-field bubbles computation:

- Bubble 1: $r_{1}(0)=0.01, z_{1}(0)=0, r_{1}(L)=0, L=2, \frac{\Delta p_{1}}{\sigma_{1}}=0.2$, 
- Bubble 2: $r_{2}(0)=0.01, z_{2}(0)=0, r_{2}(L)=0, L=2, \frac{\Delta p_{2}}{\sigma_{2}}=0.4$.

- Bubble 3: $r_{3}(0)=0.01, z_{3}(0)=0, r_{3}(L)=0, L=2, \frac{\Delta p_{3}}{\sigma_{3}}=0.6$.

- Bubble 4: $r_{4}(0)=0.01, z_{4}(0)=0, r_{4}(L)=0, L=2, \frac{\Delta p_{4}}{\sigma_{4}}=0.8$.

- Bubble 5: $r_{5}(0)=0.01, z_{5}(0)=0, r_{5}(L)=0, L=2, \frac{\Delta p_{5}}{\sigma_{5}}=1.0$.

- Bubble 6: $r_{6}(0)=0.01, z_{6}(0)=0, r_{6}(L)=0, L=2, \frac{\Delta p_{6}}{\sigma_{6}}=1.2$.

- Bubble 7: $r_{7}(0)=0.01, z_{7}(0)=0, r_{7}(L)=0, L=2, \frac{\Delta p_{7}}{\sigma_{7}}=1.4$.

- Bubble 8: $r_{8}(0)=0.01, z_{8}(0)=0, r_{8}(L)=0, L=2, \frac{\Delta p_{8}}{\sigma_{8}}=1.6$.

- Bubble 9: $r_{9}(0)=0.01, z_{9}(0)=0, r_{9}(L)=0, L=2, \frac{\Delta p_{9}}{\sigma_{9}}=1.8$.

- Bubble 10: $r_{10}(0)=0.01, z_{10}(0)=0, r_{10}(L)=0, L=2, \frac{\Delta p_{10}}{\sigma_{10}}=2.0$.

- Output-parameters of the near-field bubble computation:

- $\quad$ Bubble 1: $a_{\text {bubble }_{1}}=0.5141, b_{\text {bubble }_{1}}=0.9926$.

- Bubble 2: $a_{\text {bubble }_{2}}=0.5219, b_{\text {bubble }}=0.9718$.

- Bubble 3: $a_{\text {bubble }_{3}}=0.5295, b_{\text {bubble }}=0.9506$.

- Bubble 4: $a_{\text {bubble }_{4}}=0.5369, b_{\text {bubble }_{4}}=0.9289$.

- Bubble 5: $a_{\text {bubble }_{5}}=0.5443, b_{\text {bubble }}=0.9067$.

- Bubble 6: $a_{\text {bubble }_{6}}=0.5514, b_{\text {bubble }_{6}}=0.8841$.

- Bubble 7: $a_{\text {bubble }_{7}}=0.5584, b_{\text {bubble }_{7}}=0.8612$.

- Bubble 8: $a_{\text {bubble }}=0.5651, b_{\text {bubble }}=0.8382$.

- Bubble 9: $a_{\text {bubble }_{9}}=0.5717, b_{\text {bubble }_{9}}=0.8154$.

- Bubble 10: $a_{\text {bubble }_{10}}=0.5780, b_{\text {bubble }_{10}}=0.7928$.

Appendix A.2. Parameters of Sections 6.6 and 6.7

- Input-parameters of the near-field bubbles computation:

- Bubble 1: $r_{1}(0)=0.01, z_{1}(0)=0, r_{1}(L)=0, L=2, \frac{\Delta p_{1}}{\sigma_{1}}=0.2$,

- Bubble 2: $r_{2}(0)=0.01, z_{2}(0)=0, r_{2}(L)=0, L=2, \frac{\Delta p_{2}}{\sigma_{2}}=0.4$.

- Bubble 3: $r_{3}(0)=0.01, z_{3}(0)=0, r_{3}(L)=0, L=2, \frac{\Delta p_{3}}{\sigma_{3}}=0.6$.

- Bubble 4: $r_{4}(0)=0.01, z_{4}(0)=0, r_{4}(L)=0, L=2, \frac{\Delta p_{4}}{\sigma_{4}}=0.8$.

- Bubble 5: $r_{5}(0)=0.01, z_{5}(0)=0, r_{5}(L)=0, L=2, \frac{\Delta p_{5}}{\sigma_{5}}=1.0$.

- Bubble 6: $r_{6}(0)=0.01, z_{6}(0)=0, r_{6}(L)=0, L=2, \frac{\Delta p_{6}}{\sigma_{6}}=1.2$.

- Bubble 7: $r_{7}(0)=0.01, z_{7}(0)=0, r_{7}(L)=0, L=2, \frac{\Delta p_{7}}{\sigma_{7}}=1.4$.

- Bubble 8: $r_{8}(0)=0.01, z_{8}(0)=0, r_{8}(L)=0, L=2, \frac{\Delta p_{8}}{\sigma_{8}}=1.6$.

- Bubble 9: $r_{9}(0)=0.01, z_{9}(0)=0, r_{9}(L)=0, L=2, \frac{\Delta p_{9}}{\sigma_{9}}=1.8$.

- Bubble 10: $r_{10}(0)=0.01, z_{10}(0)=0, r_{10}(L)=0, L=2, \frac{\Delta p_{10}}{\sigma_{10}}=2.0$.

- Output-parameters of the near-field bubble computation (formation):

- $\quad$ Bubble 1: $a_{\text {bubble }_{1}}=0.5141, b_{\text {bubble }_{1}}=0.9926$.

- Bubble 2: $a_{\text {bubble }_{2}}=0.5219, b_{\text {bubble }_{2}}=0.9718$.

- Bubble 3: $a_{\text {bubble }}=0.5295, b_{\text {bubble }}=0.9506$.

- Bubble 4: $a_{\text {bubble }_{4}}=0.5369, b_{\text {bubble }_{4}}=0.9289$.

- Bubble 5: $a_{\text {bubble }_{5}}=0.5443, b_{\text {bubble }_{5}}=0.9067$.

- Bubble 6: $a_{\text {bubble }_{6}}=0.5514, b_{\text {bubble }_{6}}=0.8841$.

- Bubble 7: $a_{\text {bubble }_{7}}=0.5584, b_{\text {bubble }_{7}}=0.8612$. 
- Bubble 8: $a_{\text {bubble }}=0.5651, b_{\text {bubble }}=0.8382$.

- Bubble 9: $a_{\text {bubble } 9}=0.5717, b_{\text {bubbleg }}=0.8154$.

- Bubble 10: $a_{\text {bubble }_{10}}=0.5780, b_{\text {bubble }_{10}}=0.7928$.

- Output-parameters of the near-field bubble computation (in the E-field):

- $\quad$ Bubble 1: $a_{\text {bubble }_{1}}=0.5558, b_{\text {bubble }_{1}}=0.8698$.

- Bubble 2: $a_{\text {bubble }_{2}}=0.5626, b_{\text {bubble }_{2}}=0.8468$.

- Bubble 3: $a_{\text {bubble }_{3}}=0.5693, b_{\text {bubble }}=0.8239$.

- Bubble 4: $a_{\text {bubble }_{4}}=0.5757, b_{\text {bubble }_{4}}=0.8013$.

- Bubble 5: $a_{\text {bubble }_{5}}=0.5819, b_{\text {bubble }_{5}}=0.7789$.

- Bubble 6: $a_{\text {bubble }_{6}}=0.5878, b_{\text {bubble }_{6}}=0.7571$.

- Bubble 7: $a_{\text {bubble }}=0.5935, b_{\text {bubble }}=0.7359$.

- Bubble 8: $a_{\text {bubble }}=0.5990, b_{\text {bubble }_{8}}=0.7153$.

- Bubble 9: $a_{\text {bubble } 9}=0.6042, b_{\text {bubble }}=0.6953$.

- Bubble 10: $a_{\text {bubble }_{10}}=0.6091, b_{\text {bubble }_{10}}=0.6760$.

\section{References}

1. Gu, H.; Duits, M.H.G.; Mugele, F. Droplets formation and merging in two-phase flow microfluidics. Int. J. Mol. Sci. 2011, 12, 2572-2597. [CrossRef] [PubMed]

2. Hayashi, T.; Uehara, S.; Takana, H.; Nishiyama, H. Gas-liquid two-phase chemical reaction model of reactive plasma inside a bubble for water treatment. In Proceeding of the 22nd International Symposium on Plasma Chemistry, Antwerp, Belgium, 5-10 July 2015.

3. Kumar, R.; Kuloor, N.R. The formation of bubbles and drops. Adv. Chem. Eng. 1970, 8, 255-368.

4. Yang, G.Q.; Du, B.; Fan, L.S. Bubble formation and dynamics in gas-liquid-solid fluidization-A review. Chem. Eng. Sci. 2007, 62, 2-27. [CrossRef]

5. Ruzicka, M. Electrorheological Fluids: Modeling and Mathematical Theory; Lecture Notes in Mathematics; Springer: Berlin/Heidelberg, Germany, 2008; Volume 1748.

6. Westerterp, K.R.; Swaaij, W.P.M.V.; Beenackers, A.A.C.M. Chemical Reactor Design and Operation, 2nd ed.; John Wiley and Sons: Chichester, UK; New York, NY, USA, 1984.

7. Bruggeman, P.; Leys, C. Non-thermal plasmas in and in contact with liquids. J. Phys. D Appl. Phys. 2009, 42, 053001. [CrossRef]

8. Besagni, G.; Inzoli, F.; Ziegenhein, T. Two-phase bubble columns: A comprehensive review. ChemEngineering 2018, 2, 13. [CrossRef]

9. Mutabazi, I.; Yoshikawa, H.N.; Fogaing, M.T.; Travnikov, V.; Crumeyrolle, O.; Futterer, B.; Egber, C. Thermo-electro-hydrodynamic convection under microgravity: A review. Fluid Dyn. Res. 2016, 48, 061413. [CrossRef]

10. Sommers, B.S.; Foster, J.E. Nonlinear oscillations of gas bubbles submerged in water: Implications for plasma breakdown. J. Phys. D Appl. Phys. 2012, 45, 415203. [CrossRef]

11. Thoroddsen, S.T.; Takehara, K.; Etoh, T.G. The coalescence speed of a pendent and a sessile drop. J. Fluid Mech. 2005, 527, 85-114. [CrossRef]

12. Geiser, J.; Arab, M. Porous Media Based Modeling of PE-CVD Apparatus: Electrical fields and Deposition Geometries. In Special Topics and Reviews in Porous Media; Begell House Inc.: Redding, CA, USA, 2010; Volume 1, pp. 215-229.

13. Sethian, J.A. Level Set Methods: Evolving Interfaces in Geometry, Fluid Mechanics, Computer Vision, and Materials Science; Cambridge University Press: Cambridge, UK, 1996.

14. Hockney, R.; Eastwood, J. Computer Simulation Using Particles; CRC Press: Boca Raton, FL, USA, 1985.

15. Lapenta, G. DEMOCRITUS: An adaptive particle in cell (PIC) code for object-plasma interactions. J. Comput. Phys. 2011, 230, 4679-4695. [CrossRef]

16. Simmons, J.A.; Shikhmurzaev, J.E.S.a.Y.D. The formation of a bubble from a submerged orifice. Eur. J. Mech. B Fluids 2015, 53 (Suppl. C), 24-36. [CrossRef] 
17. Hirt, C.W.; Nichols, B.D. Volume of fluid (VOF) method for the dynamics of free boundaries. J. Comput. Phys. 1981, 39, 201-225. [CrossRef]

18. Tsui, Y.-Y.; Liu, C.-Y.; Lin, S.-W. Coupled level-set and volume-of-fluid method for two-phase flow calculations. Numer. Heat Transf. Part B Fundam. 2017, 71, 173-185. [CrossRef]

19. Harten, A.; Chakravarthy, S.R. Multi-Dimensional ENO Schemes for General Geometries; USA, NASA Contractor Report 187637; ICASE Report No. 91-76; NASA Langeley Research Center: Hamption, VA, USA, 1991; Available online: https:/ / ntrs.nasa.gov/search.jsp?R=19920001131 (accessed on 4 November 2019).

20. Hu, C.; Shu, C.-W. Weighted essentially non-oscillatory schemes on triangular meshes. J. Comput. Phys. 1999, 150, 97-127. [CrossRef]

21. Babaeva, N.Y.; Bhoj, A.N.; Kushner, M.J. Streamer dynamics in gases containing dust particles. Plasma Sources Sci. Technol. 2006, 15, 591-602. [CrossRef]

22. Kushner, M.J. Modelling of microdischarge devices: Plasma and gas dynamics. J. Phys. D Appl. Phys. 2005, 38, 1633-1643. [CrossRef]

23. Babaeva, N.Y.; Naidis, G.V.; Tereshonok, D.V.; Smirnov, B.M. Streamer breakdown in elongated, compressed and tilted bubbles immersed in water. J. Phys. D Appl. Phys. 2017, 50, 364001. [CrossRef]

24. Brennen, C.E. Cavitation and Bubble Dynamics; Oxford University Press: New York, NY, USA; Oxford, UK, 1995.

25. Geiser, J. Multicomponent and Multiscale Systems: Theory, Methods, and Applications in Engineering; Springer: Cham, Switzerland; Heidelberg, Germany; New York, NY, USA; Dordrecht, The Netherland; London, UK, 2016.

26. Osher, S.; Sethian, J.A. Fronts propagating with curvature-dependent speed: Algorithms based on Hamilton-Jacobi formulations. J. Comput. Phys. 1988, 79, 12-49. [CrossRef]

27. Stratton, J.A. Electrodynamics Theory; Mc Graw Hill: New York, NY, USA, 1941; Chapter 3.

28. Finn, R. Equilibrium Capillary Surfaces; Springer: New York, NY, USA, 1986.

29. Vafaei, S.; Wen, D. Modification of the Young-Laplace equation and prediction of bubble interface in the presence of nanoparticles. Adv. Colloid Interface Sci. 2015, 225, 1-15. [CrossRef]

30. Liu, L.; Russell, R.D. Linear system solver for boundary value ODEs. J. Comput. Appl. Math. 1993, 45, 103-117. [CrossRef]

31. Osborne, M.R. On shooting methods for boundary value problems. J. Math. Anal. Appl. 1969, 27, 417-433. [CrossRef]

32. Verführt, R. Numerical Methods and Stochastics, Part I: Numerical Methods; Lecture Notes Summerterm; Intitute of Mathematics, Ruhr-Unviersity of Bochum: Bochum, Germany, 2011.

33. Bothe, D.; Koebe, M.; Wielage, K.; Prüss, J.; Warnecke, H.-J. Direct numerical simulation of mass transfer between rising gas bubbles and water. In Bubbly Flows: Analysis, Modelling and Calculation; Sommerfeld, M., Ed.; Springer: Berlin/Heidelberg, Germany, 2004; pp. 159-174.

34. Leighton, T.G. The Acoustic Bubble; Academic Press: London, UK; San Diego, CA, USA, 1994.

35. Weinan, E. Principle of Multiscale Modelling; Cambridge University Press: Cambridge, UK, 2010.

36. Innocenti, M.E.; Lapenta, G.; Markidis, S.; Beck, A.; Vapirev, A. A multi level multi domain method for particle in cell plasma simulations. J. Comput. Phys. 2013, 238, 115-140. [CrossRef]

37. Geiser, J.; Mertin, P. Modelling approach of a near-far-field model for bubble formation and transport. arXiv 2018, arxiv:1809.00899.

38. Marmur, A.; Rubin, E. A theoretical model for bubble formation at an orifice submerged in an inviscid liquid. Chem. Eng. Sci. 1976, 31, 453-463. [CrossRef]

39. Bellini, T.; Corti, M.; Gelmetti, A.; Lago, P. Interferometric study of selectively excited bubble capillary modes. Europhys. Lett. 1997, 38, 521-526. [CrossRef]

40. Kröner, D. Numerical Schemes for Conservation Laws; Wiley-Teubner Series Advances in Numerical Mathematics; Wiley-Teubner: Chichester, UK, 1997.

41. Geiser, J.; Buck, V.; Arab, M. Model of PE-CVD Apparatus: Verification and Simulations. Math. Prob. Eng. 2010, 2010, 407561. [CrossRef]

(C) 2019 by the authors. Licensee MDPI, Basel, Switzerland. This article is an open access article distributed under the terms and conditions of the Creative Commons Attribution (CC BY) license (http:/ / creativecommons.org/licenses/by/4.0/). 